\title{
Influence of redox processes on clay mineral transformation in Retisols in the Carpathian Foothills in Poland. Is a ferrolysis process present?
}

\author{
Wojciech Szymański $^{1} \cdot$ Michal Skiba $^{2} \cdot$ Artur Blachowski $^{3}$
}

Received: 16 May 2016 / Accepted: 18 August 2016/Published online: 9 September 2016

(C) The Author(s) 2016. This article is published with open access at Springerlink.com

\begin{abstract}
Purpose Ferrolysis is a soil-forming process, which involves destruction of clay minerals due to cyclic reduction and oxidation in acidic and periodically wet soils. The main objectives of this study were as follows: (1) to determine the influence of redox processes on clay mineral transformation in Retisols (Albeluvisols) in the Carpathian Foothills in Poland and (2) to verify the occurrence of ferrolysis in Retisols showing various degrees of bleaching.

Materials and methods Twelve representative soil profiles were selected for analysis. All were formed entirely from loess except for two profiles, in which the lowermost horizons (2C) had developed from weathered flysch rocks residuum. Soil mineral analysis was done using x-ray diffraction (XRD), Fourier-transform infrared (FTIR) spectroscopy, and Mössbauer spectroscopy (MS).

Results and discussion The obtained results indicate that the qualitative and quantitative mineral compositions of the clay fraction in the E and Eg horizons obtained from Retisols in the Carpathian Foothills exhibiting marked
\end{abstract}

Responsible editor: Dong-Mei Zhou

Wojciech Szymański

w.szymanski@uj.edu.pl

1 Institute of Geography and Spatial Management, Department of Pedology and Soil Geography, Jagiellonian University, ul. Gronostajowa 7, 30-387 Kraków, Poland

2 Institute of Geological Sciences, Department of Mineralogy, Petrology and Geochemistry, Jagiellonian University, ul. Oleandry 2a, 30-063 Kraków, Poland

3 Institute of Physics, Department of Mössbauer Spectroscopy, Pedagogical University, ul. Podchorążych 2, 30-084 Kraków, Poland differences in bleaching (strong, moderate, weak, and lack of bleaching) caused by periodic stagnation of water above a slowly permeable fragipan and cyclic redox processes are the same. The E and Eg horizons are characterized by the presence of 2:1 clay minerals with likely organic interlayer fillings, dioctahedral mica, kaolinite, and chlorite.

Conclusions The results indicate that (1) redox processes occurring in the soils do not affect clay mineral transformation in Retisols of the Carpathian Foothills in Poland and (2) ferrolysis is not the main soil-forming process operating in these soils. This is most likely because ironbearing minerals are not abundant in the Retisols and/or undergo eluviation to the lower part of the soil profiles. The lower content of the clay fraction in the $\mathrm{E}$ and $\mathrm{Eg}$ horizons versus that in the lower soil horizons of the Retisols is related to clay illuviation (lessivage), and not to clay decomposition due to ferrolysis.

Keywords Carpathian Foothills · Clay minerals · Ferrolysis · Redox processes $\cdot$ Retisols $\cdot \mathrm{X}$-ray diffraction

\section{Introduction}

Soils with a contrasting texture are very common around the world (Kemp and McIntosh 1989; Phillips 2001, 2004, 2007; Świtoniak 2008, 2014; Świtoniak et al. 2016). According to the literature, such soils could be the result of different geologic and/or pedologic processes including sedimentation of different parent material (Phillips 2007; Świtoniak 2008, 2014; Świtoniak et al. 2016); clay eluviation-illuviation (Van Ranst and De Coninck 2002; Boivin et al. 2004; Świtoniak 2008, 2014; Świtoniak et al. 2016); bioturbation (Leigh 1998; Peacock and Fant 
2002; Phillips 2007); and ferrolysis (Brinkman 1970, 1977, 1979; Brinkman et al. 1973).

Ferrolysis is a soil-forming process, which was first proposed by Brinkman (1970). It involves destruction of clay minerals due to cyclic reduction and oxidation in acidic and periodically wet soils (Brinkman 1970; Barbiero et al. 2010). Ferrolysis involves biochemical reduction of $\mathrm{Fe}^{3+}$ taking place during wet seasons and leading to mobilization of $\mathrm{Fe}$ and substitution of exchangeable cations within clay minerals with $\mathrm{Fe}^{2+}$. During dry periods, $\mathrm{Fe}^{2+}$ oxidizes forming ferric hydroxides and protons which attacks structures of clay minerals. Thus, cyclic reduction and oxidation may cause decomposition of clay minerals leading to release of the lattice cations (e.g., $\mathrm{Al}, \mathrm{Mg}, \mathrm{Fe}$ ). Except for the dissolution, soil clay minerals may undergo transformation due to the formation of Al- and/or Fehydroxy polymers within the interlayer space (Rich 1968; Brinkman 1970, 1977; Gerstl and Banin 1980). The transformation of 2:1 clay minerals due to the cyclic reduction and oxidation and formation of the clays with interlayer fillings is responsible for lowering their cation exchange capacity, mineral solubility, and swelling ability (Rich 1968; Brinkman 1979; Nakao et al. 2009; Szymański et al. 2014).

Ferrolysis is a common process in seasonally wet and acidic soils containing a hardly permeable horizon such as a plughpan (Brinkman 1977) and duripan (Hobson and Dahlgren 1998). Ferrolysis may also occur in soils with a fragipan. Fragipan shows low permeability which may lead to periodic water stagnation causing seasonal anaerobic conditions which favor reduction of iron oxides and their subsequent translocation and oxidation. Thus, it is very likely that ferrolysis may take place in Retisols containing a fragipan, because of the high bulk density and low porosity of the pan (e.g., Witty and Knox 1989; Lindbo et al. 1994; Ciolkosz et al. 1995; Szymański et al. 2011). The seasonal stagnation of water within Retisols causes the bleaching of soil material indicating reduction and dissolution of iron oxides and their subsequent re-oxidation and immobilization in the form of iron nodules, concretions, soft masses, and pore linings (Cescas et al. 1970; Schwertmann and Fanning 1976; Rhoton et al. 1993; Zhang and Karathanasis 1997; Lindbo et al. 2000; Dixon and White 2002; Szymański et al. 2011, 2012, 2014; Szymański and Skiba 2013). Similar changes in the valence of iron may occur within the structures of clay minerals containing iron in the octahedral sheet (e.g., Rich 1968; Brinkman 1977; Rozenson and Heller-Kallai 1978; Gerstl and Banin 1980; Hardy et al. 1999; Kohut and Warren 2002). However, Van Ranst and De Coninck (2002) as well as Van Ranst et al. (2011) have recently reported that ferrolysis does not take place in Retisols (previously called Albeluvisols) and soils exhibiting a stagnic color pattern from Western Europe and in Planosols from Ethiopia.

Despite the fact that the redox processes are very common in various soils (Retisols, Stagnosols, Planosols, Gleysols, Fluvisols, paddy soils), very few is known about their impact on soil minerals and especially on clay minerals. In addition, very little studies present the results of qualitative and quantitative mineral composition of hydromorphic soils in the context of their genesis. Due to the above-mentioned contrasting results and reports concerning ferrolysis process in soil environment and still lacking of sufficient detailed mineralogical data from hydromorphic soils, we have decided to fill this gap.

The main objectives of this study were the following: (1) to determine the influence of redox processes on clay mineral transformation in Retisols (Albeluvisols) in the Carpathian Foothills in Poland and (2) to verify the occurrence of ferrolysis in Retisols showing various degrees of bleaching by means of qualitative and quantitative mineralogical analyses.

\section{Materials and methods}

\subsection{Study area}

The present study was carried out in the Carpathian Foothills in southern Poland (Fig. 1). The region is formed of a thick mantle of non-calcareous loess, which covers interbedded layers of sandstone, siltstone, and shale of turbiditic origin (i.e., Carpathian flysch). According to Klimaszewski (1967), the loess was transported on a short distance and accumulated during the last phase of glaciation (Vistulian) in the Pleistocene. The studied area is characterized by hilly relief with wide and flat hills showing elevation between 300 and $500 \mathrm{~m}$ a.s.l. (Starkel 1972). A moderately humid climate with mean annual air temperature between 6 and $8{ }^{\circ} \mathrm{C}$ and total annual precipitation from 700 to $900 \mathrm{~mm}$ (Hess 1965) as well as a lack of carbonates in the loess cover serve as the main reasons for the prevalence of Luvisols and Retisols (which until recently were called Albeluvisols) across the Carpathian Foothills (Skiba and Drewnik 2003; Szymański et al. 2011, 2012). The vegetation of the study area can be characterized by a mosaic of broadleaf forests (TilioCarpinetum) showing a prevalence of hornbeam (Carpinus betulus L.), lime (Tilia cordata Mill.), oak (Quercus sessilis Ehrh. and Quercus robur L.), and beech (Fagus sylvatica L.) as well as arable land, with potatoes (Solanum tuberosum L.), wheat (Triticum aestivum L.), and rye (Secale cereale) being the most popular crops. In some places, mixed forests (PinoQuercetum) with oak (Q. robur L.) and pine (Pinus silvestris L.) are also present (Towpasz and Zemanek 1995). 


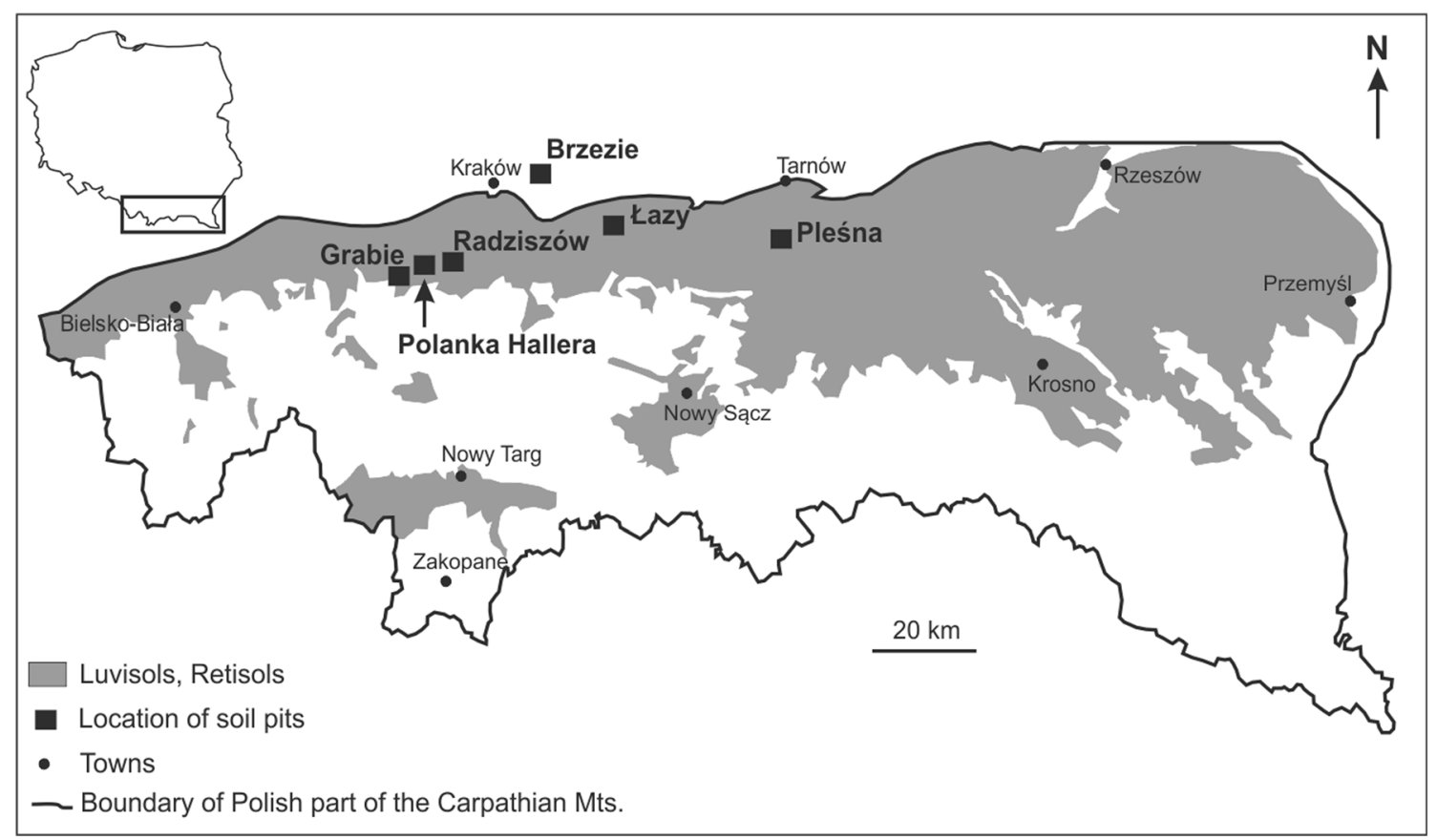

Fig. 1 Location of the soil profiles and occurrence of Luvisols and Retisols in the Polish part of the Carpathians (based on Skiba and Drewnik 2003)

\subsection{Methods}

Twelve representative soil profiles were selected for analysis. All were formed entirely from loess except for the Grabie and Radziszów profiles, in which the lowermost horizons $(2 \mathrm{C})$ had developed from weathered flysch rocks residuum. Figure 1 presents the geographic location of the soil profiles, while Table 1 summarizes detailed information about the study sites and classification of the soil profiles according to the World Reference Base for Soil Resources (WRB) system (IUSS Working Group WRB 2014).

During field studies, soil profiles were described and sampled. Soil samples were collected from each soil horizon in plastic bags and transported to the laboratory. In the laboratory, the samples were air dried, gently crushed, and sieved through a 2-mm sieve. All laboratory analyses were conducted on the fraction below $2 \mathrm{~mm}$. Texture of soil material was determined by means of a set of sieves for the purpose of sand separation as well as the hydrometer method for silt and clay fractions (Gee and Bauder 1986). The content of organic carbon was determined using a rapid dichromate oxidation technique (Nelson and Sommers 1996), while $\mathrm{pH}$ was measured potentiometrically in distilled water using a 1:2.5 soil/water ratio $(w / v)$ (Thomas 1996). Chemical composition of the fine-earth material (fraction $<2 \mathrm{~mm}$ ) was determined using inductively coupled plasma-emission spectrometry (ICP-ES) following digestion of the bulk samples with lithium metaborate/tetraborate and dilute nitric acid at Acme Labs (Vancouver, BC, Canada). Free forms of iron $\left(\mathrm{Fe}_{\mathrm{d}}\right)$ extracted using dithionite-citratebicarbonate (DCB) were determined by means of atomic absorption spectrometry (AAS) (Schlichting and Blume 1966). Amorphous forms of iron and aluminum $\left(\mathrm{Fe}_{\mathrm{o}}, \mathrm{Al}_{\mathrm{o}}\right.$, respectively) were extracted with acid ammonium oxalate (Van Reeuwijk 2002) and determined using a spectrophotometer (Rayleigh UV-1601). The color of the soil material was described in the moist state using Munsell Color Soil Charts (where, e.g., 10YR means hue and 6/2 means value/ chroma of color).

X-ray diffraction analyses were done using a Philips X'Pert diffractometer equipped with a PW3020 vertical goniometer, $1^{\circ}$ divergence slit, $0.2 \mathrm{~mm}$ receiving slit, incident- and diffracted-beam Soller slits, $1^{\circ}$ anti-scatter slit, graphite diffracted-beam monochromator, and a $\mathrm{Cu}$ x-ray lamp operated at $40 \mathrm{kV}$ and $30 \mathrm{~mA}$.

Quantitative analyses were performed on bulk soil samples ( $<2 \mathrm{~mm}$ ) mixed with $\mathrm{ZnO}$ as internal standard using random powder mounts (Moore and Reynolds 1997). More details concerning the preparation of samples for quantitative analysis of the mineral composition can be found in Szymański et al. (2011) and Drewnik et al. (2014). Random specimens were scanned from 2 to $65^{\circ} 2 \Theta$ at a counting speed of $0.02^{\circ}$ \% $5 \mathrm{~s}$. To calculate the mineral composition of the bulk samples, the Seifert Rietveld AutoQuan/BGMN software was used (Taut et al. 1998). For calculation purposes, X-ray diffraction (XRD) data from $15^{\circ} 2 \Theta$ to $65^{\circ} 2 \Theta$ were used.

Clay fractions $(<2 \mu \mathrm{m})$ were separated by centrifugation (using a centrifuge MPW-400) of $100 \mathrm{~g}$ bulk samples $(<2 \mathrm{~mm}$ ) dispersed in deionized water using an ultrasonic disintegrator. The centrifugation time was $3 \mathrm{~min}$ at the speed of $1000 \mathrm{rpm}$ and the assumed density of particles was $2.65 \mathrm{Mg} / \mathrm{m}^{3}$ (Jackson 1969). To obtain clay minerals in a natural state, the samples 


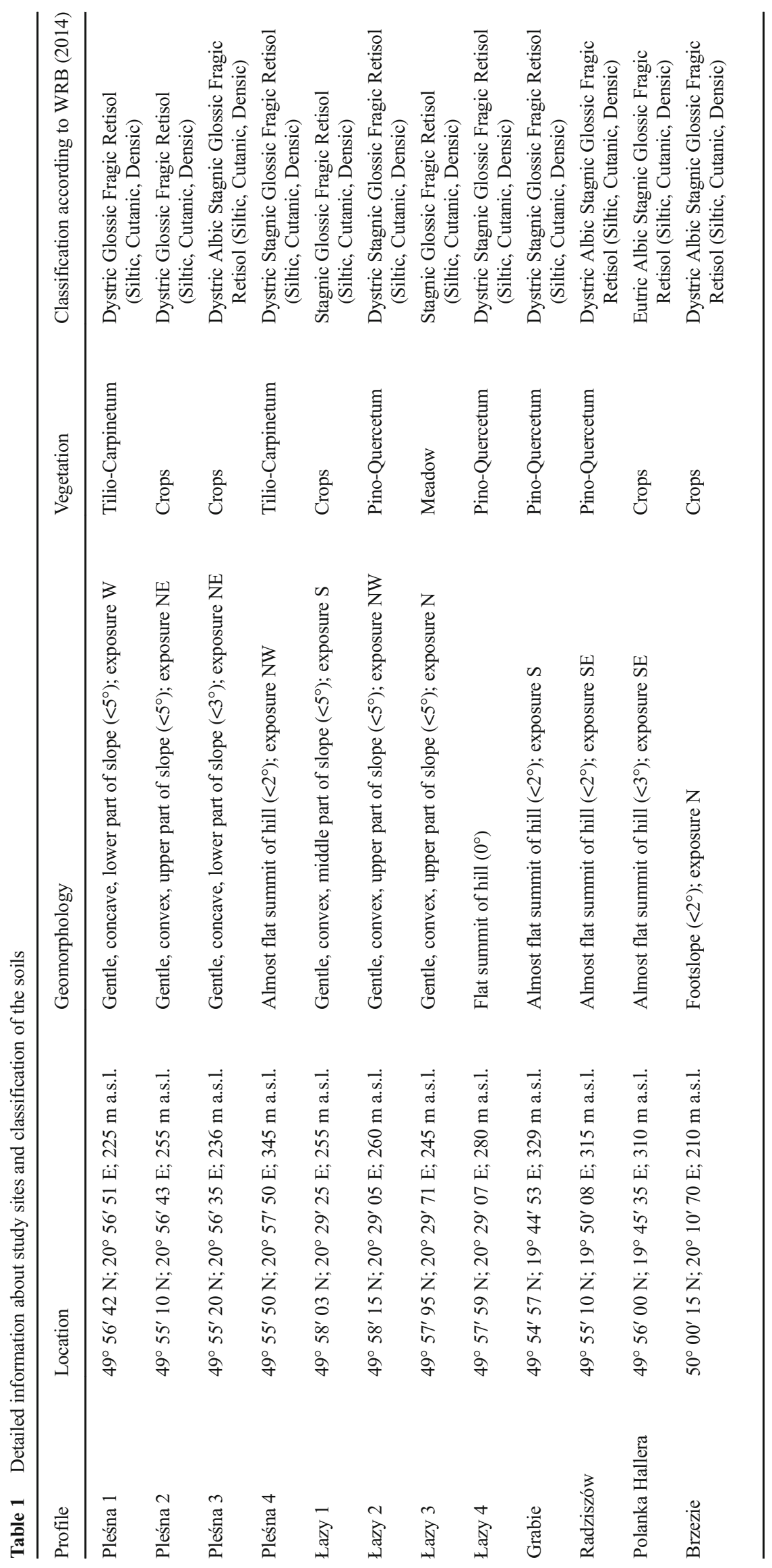


were separated without any chemical pretreatment. Oriented mounts with a surface density of $10 \mathrm{mg} / \mathrm{cm}^{2}$ were prepared from the separated clay fractions by means of a glass slide technique (Środoń, 2006). XRD patterns for the oriented specimens were registered in air dry state, after heating for $1 \mathrm{~h}$ at 330 and at $550{ }^{\circ} \mathrm{C}$, and after solvation, the mounts with liquid glycerol. The identification of clay minerals was performed according to operational definitions given by Środoń (2006). Analyses of XRD patterns were conducted by means of a ClayLab software (Mystkowski 1999).

Fourier-transform infrared (FTIR) spectra for the clay fractions were collected for random powder specimens dispersed in $\mathrm{KBr}$ pellets using a Bio-Rad FTS 135 FTIR spectrometer. The pellets (1.6 mg sample/300 mg KBr) were prepared from the natural clay fraction $(<2 \mu \mathrm{m})$ that was previously dried by heating at $105{ }^{\circ} \mathrm{C}$ for $24 \mathrm{~h}$ and from $\mathrm{KBr}$ powder that was preheated at $550{ }^{\circ} \mathrm{C}$ for $24 \mathrm{~h}$. Following the collection of the FTIR spectra, the pellets were heated at $180^{\circ} \mathrm{C}$ for $12 \mathrm{~h}$, and after cooling in a desiccator, analyzed again. Thirty-two scans were collected for each sample in the range from 400 to $4000 \mathrm{~cm}^{-1}$ with a resolution of $2 \mathrm{~cm}^{-1}$. The FTIR spectra were processed using a Spekwin32 software.

Mössbauer spectroscopy measurements were performed at room temperature using a RENON MsAa-3 spectrometer equipped with an LND Kr-filled proportional detector and He-Ne laser-based interferometer used to calibrate a velocity scale. A commercial ${ }^{57} \mathrm{Co}(\mathrm{Rh})$ source was applied for the $14.41-\mathrm{keV}$ resonant transition in ${ }^{57} \mathrm{Fe}$. The absorber for Mössbauer measurements was prepared in powder form and the thickness of the absorber was $90 \mathrm{mg} / \mathrm{cm}^{2}$ of the investigated material. Data were fitted following the transmission integral approach using the MOSGRAF software suite.

Differences in mineralogical composition were evaluated by $t$ Student test (level of significance at $p<0.05$ ). All statistical calculations were done using Statistica 12 software.

\section{Results and discussion}

\subsection{Morphology, physical, and chemical properties}

The morphologic description of the soil profiles is summarized in Table 2. All the soil profiles show similar morphology, i.e., A-E-Btx-Btg-BC-C. The two main differences between the soils are the occurrence of organic horizons $(\mathrm{O})$ in forest soils and strong (Pleśna 3, Pleśna 4, Łazy 1, Polanka Hallera, and Brzezie profiles); moderate (Łazy 3, Łazy 4, Grabie, and Radziszów profiles); or very weak or even nonexistent (Pleśna 1, Pleśna 2, and Łazy 2 profiles) bleaching of the eluvial part of the profile. The bleaching classification was done according to Munsell soil color charts in moist state: $10 \mathrm{YR} 6 / 2$; 10YR 7/2-8/2, 2.5Y 6/2 (strongly bleached soil horizons); 10YR 4/3-5/3 (moderately bleached soil horizons); and
10YR 5/4 (very weakly or not bleached soil horizons). In addition, Pleśna 4, Łazy 4, and Radziszów profiles were further characterized by a bipartite eluvial horizon with an upper part showing lack of bleaching and a lower part (along the contact with the lower illuvial horizon) exhibiting moderate or strong bleaching. Topsoil horizons show black or brownish color, lack of Fe-Mn nodules and clay coatings on ped faces. Eluvial horizons (AE, E, Eg) exhibit more pale and/or bleached color and occurrence of hard Fe-Mn nodules. However, the Eg horizons contain more Fe-Mn nodules than the E horizons based on visual inspection of the soil profiles (Table 2). The lower part of the soil profiles usually exhibits mottled color. Additionally, the lower soil horizons are characterized by presence of many hard and soft Fe-Mn nodules, and many clay coatings on ped faces and clay infillings in channels (Table 2) (Szymański et al. 2011, 2012). Such a morphology of the soils is very typical for Retisols (Albeluvisols) occurring in different areas around the world (e.g., Brinkman 1979; Lindbo et al. 1994, 1995, 2000; Montagne et al. 2008; Kalinina et al. 2013; Sauer et al. 2013; Nikorych et al. 2014).

All the soils show a silt loam texture with a predominance of the silt fraction (Table 3). The topsoil and eluvial horizons contain markedly lower amounts of the clay fraction in comparison with lower horizons. Such a distribution of the clay fraction and the presence of many clay coatings and clay infillings clearly indicate a vertical translocation of the clay fraction in the soil profiles (Zasoński 1983, 1992; Lindbo and Veneman 1993; Weisenborn and Schaetzl 2005; Szymański et al. 2011, 2012). The lower part of the soil is characterized by higher bulk density and lower total porosity than those of the upper soil horizons (Table 3). The lower bulk density and higher total porosity of the upper soil horizons relative to lower soil horizons also indicate depletion of the finest particles and their accumulation in the lower part of the soils (Szymański et al. 2011, 2012, 2014; Nikorych et al. 2014).

The majority of the soils exhibit acidic or slightly acidic $\mathrm{pH}$ and only the Polanka Hallera profile is characterized by a slightly alkaline $\mathrm{pH}$ in some soil horizons, which is due to the liming.

Soil organic carbon (SOC) is concentrated mainly in topsoil horizons and its content in the horizons of forest soils ranges from $1.2 \%$ to $6.1 \%$ and is markedly higher than SOC content in topsoil horizons of arable soils ( $0.7 \%$ to 1.2 $\%)$. Eluvial and illuvial soil horizons contain a very low amount of SOC $(0.1 \%$ to $0.7 \%)$ (Table 3$)$.

The content of DCB-extractable iron $\left(\mathrm{Fe}_{\mathrm{d}}\right)$ and AAOextractable iron and aluminum $\left(\mathrm{Fe}_{\mathrm{o}}\right.$ and $\mathrm{Al}_{\mathrm{o}}$, respectively) is shown in Table $3 . \mathrm{Fe}_{\mathrm{d}}$ content is lower (statistically significant at $p<0.05)$ in topsoil and eluvial horizons $(3.73$ to $9.02 \mathrm{~g} \mathrm{~kg}$ ${ }^{-1}$ with a mean of $5.22 \mathrm{~g} \mathrm{~kg}^{-1}$ ) than in lower horizons (Btx, $\mathrm{Btg}, \mathrm{BC}, \mathrm{C}$ ) (4.35 to $17.83 \mathrm{~g} \mathrm{~kg}^{-1}$ with a mean of $7.87 \mathrm{~g} \mathrm{~kg}^{-1}$ ), indicating translocation of $\mathrm{Fe}_{\mathrm{d}}$ with clay minerals down the 
Table 2 Field description of morphology of the soils

\begin{tabular}{|c|c|c|c|c|c|c|c|}
\hline Horizon & Depth $(\mathrm{cm})$ & Color (moist) & Structure & Consistence & Roots & Fe-Mn nodules & Clay coatings \\
\hline \multicolumn{8}{|l|}{ Pleśna 1} \\
\hline $\mathrm{O}$ & $0-2$ & N.A. & N.A. & N.A. & N.A. & N.A. & N.A. \\
\hline $\mathrm{A}$ & $2-10$ & $10 Y R$ 4/2 & sub- to angular blocky & soft & +++ & absence & absence \\
\hline $\mathrm{AE}$ & $10-30$ & 10YR 5/3 & sub- to angular blocky & soft & ++ & few & absence \\
\hline $\mathrm{E}$ & $30-70$ & $10 Y R$ 5/4 & sub- to angular blocky & slightly hard & + & absence & absence \\
\hline Btx & $70-105$ & $10 \mathrm{YR} 4 / 6$ & prismatic & very hard & few & +++ & +++ \\
\hline Btg & $105-150$ & 10YR 4/6 & prismatic & very hard & absence & absence & +++ \\
\hline $\mathrm{BC}$ & $150-200$ & 10YR 5/4 & massive & hard & absence & absence & absence \\
\hline \multicolumn{8}{|l|}{ Pleśna 2} \\
\hline A & $0-10$ & $10 Y R 5 / 3$ & subangular blocky & slightly hard & +++ & absence & absence \\
\hline $\mathrm{AE}$ & $10-30$ & $10 Y R 5 / 4$ & sub- to angular blocky & slightly hard & ++ & absence & absence \\
\hline $\mathrm{E}$ & $30-55$ & 10YR 5/4 & sub- to angular blocky & slightly hard & + & few & absence \\
\hline Btx1 & $55-80$ & 10YR $5 / 6 ; 10$ YR $6 / 4$ & prismatic & hard & few & +++ & +++ \\
\hline Btx2 & $80-160$ & 10 YR $5 / 6 ; 10$ YR $6 / 4$ & prismatic & very hard & absence & +++ & +++ \\
\hline \multicolumn{8}{|l|}{ Pleśna 3} \\
\hline Ap & $0-35$ & $10 Y R 4 / 3$ & angular blocky & slightly hard & +++ & absence & absence \\
\hline $\mathrm{Eg}$ & $35-45$ & $10 \mathrm{YR} 6 / 2$ & angular blocky & slightly hard & + & +++ & absence \\
\hline Btx1 & $45-100$ & 10YR 5/4; 10YR 6/2 & prismatic & very hard & few & +++ & +++ \\
\hline Btx2 & $100-180$ & 10 YR $5 / 3 ; 10$ YR $6 / 2$ & prismatic & very hard & absence & +++ & +++ \\
\hline $\mathrm{Btg}$ & $180-245$ & 10 YR $5 / 2 ; 10$ YR $6 / 2$ & massive & hard & absence & + & + \\
\hline $\mathrm{BCg}$ & $245-285$ & 10 YR $5 / 2$ & massive & hard & absence & + & + \\
\hline \multicolumn{8}{|l|}{ Pleśna 4} \\
\hline $\mathrm{O}$ & $0-2$ & N.A. & N.A. & N.A. & N.A. & N.A. & N.A. \\
\hline $\mathrm{A}$ & $2-10$ & 10YR 2/1 & sub- to angular blocky & soft & +++ & absence & absence \\
\hline $\mathrm{E}$ & $10-40$ & 10YR 5/4 & sub- to angular blocky & slightly hard & +++ & absence & absence \\
\hline $\mathrm{Eg}$ & $40-90$ & $2.5 \mathrm{Y} 6 / 2$ & angular blocky & hard & few & +++ & absence \\
\hline Btx & $90-150$ & $10 \mathrm{YR} 4 / 6 ; 2.5 \mathrm{Y} 6 / 2$ & prismatic & very hard & absence & ++ & +++ \\
\hline \multicolumn{8}{|l|}{ Łazy 1} \\
\hline Ap & $0-35$ & $10 Y R 3 / 3$ & subangular blocky & soft & +++ & absence & absence \\
\hline $\mathrm{Eg}$ & $35-50$ & 10YR 6/2 & ang- to subangular blocky & slightly hard & + & + & absence \\
\hline Btx & $50-70$ & 10YR 5/6; 10YR 8/1 & angular blocky & slightly hard & few & +++ & +++ \\
\hline Btg & $70-90$ & 10 YR $5 / 6$ & angular blocky & hard & absence & absence & +++ \\
\hline $\mathrm{BC}$ & $90-110$ & 10YR 5/4 & ang- to subangular blocky & hard & absence & absence & + \\
\hline $\mathrm{C}$ & $110-150$ & 10YR 5/4 & massive & hard & absence & absence & absence \\
\hline \multicolumn{8}{|l|}{ Lazy 2} \\
\hline $\mathrm{Ol}$ & $0-5$ & N.A. & N.A. & N.A. & N.A. & N.A. & N.A. \\
\hline Of & $5-8$ & N.A. & N.A. & N.A. & N.A. & N.A. & N.A. \\
\hline A & $8-20$ & 10YR $3 / 2$ & sub- to angular blocky & soft & +++ & absence & absence \\
\hline $\mathrm{E}$ & $20-55$ & 10YR $5 / 4$ & subangular blocky & slightly hard & ++ & absence & absence \\
\hline Btx1 & $55-65$ & 10YR 4/6 & angular blocky & hard & few & few & +++ \\
\hline Btx2 & $65-85$ & $10 Y R 4 / 6$ & angular blocky & very hard & few & few & +++ \\
\hline $\mathrm{Bt}$ & $85-115$ & $10 \mathrm{YR} 4 / 6$ & angular blocky & very hard & few & few & +++ \\
\hline $\mathrm{BC}$ & $115-135$ & $10 \mathrm{YR} 4 / 6$ & angular blocky & very hard & few & absence & ++ \\
\hline $\mathrm{C}$ & $135-185$ & $10 \mathrm{YR} 4 / 6$ & massive & very hard & absence & absence & absence \\
\hline \multicolumn{8}{|l|}{ Łazy 3} \\
\hline $\mathrm{Ol}$ & $0-3$ & N.A. & N.A. & N.A. & N.A. & N.A. & N.A. \\
\hline A & $3-11$ & 10YR $3 / 3$ & sub- to angular blocky & soft & ++ & absence & absence \\
\hline $\mathrm{AE}$ & $11-25$ & $10 Y R 4 / 3$ & sub- to angular blocky & soft & + & absence & absence \\
\hline $\mathrm{Eg}$ & $25-50$ & $10 \mathrm{YR} 4 / 3$ & sub- to angular blocky & soft & few & few & absence \\
\hline $\mathrm{Bh}$ & $50-70$ & $10 \mathrm{YR} 3 / 3 ; 10 \mathrm{YR} 2 / 3$ & sub- to angular blocky & slightly hard & few & absence & + \\
\hline Btx1 & $70-110$ & 10 YR $4 / 6 ; 10$ YR $6 / 4$ & prismatic & very hard & few & +++ & +++ \\
\hline Btx2 & $110-160$ & 10YR 5/6; 10YR 6/4 & prismatic & very hard & absence & +++ & +++ \\
\hline \multicolumn{8}{|l|}{ Łazy 4} \\
\hline $\mathrm{Ol}$ & $0-2$ & N.A. & N.A. & N.A. & N.A. & N.A. & N.A. \\
\hline $\mathrm{Ah}$ & $2-5$ & $10 \mathrm{YR} 2 / 2$ & subangular blocky to granular & soft & +++ & absence & absence \\
\hline $\mathrm{E}$ & $5-20$ & 10YR 5/4 & angular blocky & slightly hard & ++ & few & absence \\
\hline $\mathrm{Eg}$ & $20-35$ & 10 YR $5 / 3$ & angular blocky & slightly hard & + & ++ & absence \\
\hline Btx1 & $35-60$ & 10YR 5/4; 10YR 6/2 & prismatic & very hard & few & +++ & +++ \\
\hline Btx2 & $60-85$ & 10 YR $5 / 6 ; 10$ YR $6 / 2$ & prismatic & very hard & few & +++ & +++ \\
\hline Btg & $85-140$ & 10YR 5/4; 10YR 5/6 & prismatic & very hard & absence & ++ & ++ \\
\hline $\mathrm{BCg}$ & $140-180$ & 10 YR $5 / 4 ; 10$ YR $5 / 6$ & massive & hard & absence & ++ & + \\
\hline \multicolumn{8}{|l|}{ Grabie } \\
\hline $\mathrm{Ol}$ & $0-2$ & N.A. & N.A. & N.A. & N.A. & N.A. & N.A. \\
\hline Ofh & $2-4$ & N.A. & N.A. & N.A. & N.A. & N.A. & N.A. \\
\hline $\mathrm{Ah}$ & $4-12$ & 10YR $2 / 2$ & subangular blocky to granular & soft & +++ & absence & absence \\
\hline
\end{tabular}


Table 2 (continued)

\begin{tabular}{|c|c|c|c|c|c|c|c|}
\hline Horizon & Depth $(\mathrm{cm})$ & Color (moist) & Structure & Consistence & Roots & Fe-Mn nodules & Clay coatings \\
\hline $\mathrm{Eg}$ & $12-35$ & 10YR 5/3 & ang- to subangular blocky & slightly hard & ++ & ++ & absence \\
\hline Btx1 & $35-70$ & 10YR 4/6; 10YR 6/4 & prismatic & very hard & few & +++ & +++ \\
\hline Btx2 & $70-120$ & 10YR 5/6; 10YR 6/4 & prismatic & very hard & few & ++ & +++ \\
\hline Btx3 & $120-155$ & 10YR 5/6; 10YR 7/1 & prismatic & very hard & few & ++ & ++ \\
\hline $2 \mathrm{C}$ & $155-165$ & $2.5 Y 5 / 4$ & massive & very hard & few & absence & absence \\
\hline \multicolumn{8}{|c|}{ Radziszów } \\
\hline Of & $0-2$ & N.A. & N.A. & N.A. & N.A. & N.A. & N.A. \\
\hline $\mathrm{Ah}$ & $2-12$ & 10YR $2 / 2$ & granular to subangular blocky & soft & +++ & absence & absence \\
\hline $\mathrm{E}$ & $12-35$ & 10YR 5/4 & ang- to subangular blocky & slightly hard & ++ & few & absence \\
\hline $\mathrm{Eg}$ & $35-45$ & 10 YR $5 / 3$ & ang- to subangular blocky & slightly hard & + & ++ & absence \\
\hline Btx1 & $45-75$ & 10YR 5/6; 10YR 7/2 & prismatic & very hard & few & ++ & +++ \\
\hline Btx2 & $75-100$ & 10YR 5/6; 10YR 7/2 & prismatic & very hard & few & +++ & +++ \\
\hline Btx3 & $100-120$ & 10 YR 5/6; 2.5 Y 6/2 & prismatic & very hard & few & ++ & + \\
\hline $\mathrm{BC}$ & $120-130$ & 10YR 5/6; 2.5 Y 6/2 & massive & very hard & few & ++ & + \\
\hline $2 \mathrm{C}$ & $130-160$ & $2.5 \mathrm{Y} 5 / 4$ & massive & very hard & absence & absence & absence \\
\hline \multicolumn{8}{|c|}{ Polanka Hallera } \\
\hline Ap & $0-20$ & $10 Y R 3 / 3$ & subangular blocky & slightly hard & + & absence & absence \\
\hline A & $20-37$ & $10 \mathrm{YR} 3 / 3$ & sub- to angular blocky & hard & + & absence & absence \\
\hline $\mathrm{Eg}$ & $37-50$ & $10 \mathrm{YR} 8 / 2$ & subangular blocky & hard & absence & +++ & absence \\
\hline Btx & $50-60$ & 10YR 5/4; 10YR 8/2 & prismatic & very hard & absence & ++ & +++ \\
\hline Btg1 & $60-85$ & 10YR 5/4; 10YR 8/2 & prismatic & very hard & absence & ++ & +++ \\
\hline Btg2 & $85-120$ & 10YR 5/4; 10YR 8/2 & prismatic & very hard & absence & ++ & +++ \\
\hline Btg3 & $120-155$ & 10 YR $5 / 8 ; 10$ YR $7 / 3$ & prismatic & very hard & absence & + & +++ \\
\hline Btg4 & $155-180$ & 10 YR $6 / 6 ; 2.5 Y$ Y $7 / 2$ & prismatic & very hard & absence & + & ++ \\
\hline \multicolumn{8}{|l|}{ Brzezie } \\
\hline Ap & $0-25$ & 10 YR $5 / 3$ & angular blocky & slightly hard & +++ & absence & absence \\
\hline AE1 & $25-40$ & 10YR 5/4 & angular blocky & slightly hard & ++ & absence & absence \\
\hline AE2 & $40-58$ & $10 Y R$ S/3 & angular blocky to granular & slightly hard & ++ & absence & absence \\
\hline $\mathrm{Eg}$ & $58-70$ & $10 \mathrm{YR} 7 / 2$ & angular blocky to granular & slightly hard & + & +++ & absence \\
\hline Btx & $70-95$ & 10 YR $6 / 8 ; 10$ YR $7 / 2$ & prismatic & hard & few & +++ & +++ \\
\hline Btg1 & $95-115$ & 10 YR $6 / 6 ; 10$ YR 6/1 & prismatic & hard & absence & +++ & +++ \\
\hline Btg2 & $115-180$ & 10YR 6/6; 10YR 7/1 & prismatic & hard & absence & ++ & +++ \\
\hline
\end{tabular}

N.A. not analyzed

soil profile. Bleached eluvial soil horizons (Eg) exhibit a slightly higher (but not statistically significant at $p<0.05$ ) mean content of $\mathrm{Fe}_{\mathrm{d}}\left(5.33 \mathrm{~g} \mathrm{~kg}^{-1}\right)$ than other eluvial horizons (E), in which the mean content of $\mathrm{Fe}_{\mathrm{d}}$ is $4.81 \mathrm{~g} \mathrm{~kg}^{-1}$. $\mathrm{Fe}_{\mathrm{o}}$ and $\mathrm{Al}_{\mathrm{o}}$ content is slightly higher in $\mathrm{A}, \mathrm{AE}, \mathrm{E}$, and Eg horizons (with a mean of 2.62 and $0.92 \mathrm{~g} \mathrm{~kg}^{-1}$, respectively) than in lower soil horizons (with a mean of 2.13 and $0.85 \mathrm{~g} \mathrm{~kg}^{-1}$, respectively) or is evenly distributed throughout the soil profiles (Table 3). Mean $\mathrm{Fe}_{\mathrm{o}}$ content is only slightly higher (but not statistically significant at $p<0.05$ ) in $\mathrm{E}$ horizons $\left(2.57 \mathrm{~g} \mathrm{~kg}^{-1}\right)$ than in Eg horizons $\left(2.49 \mathrm{~g} \mathrm{~kg}^{-1}\right)$, while mean $\mathrm{Al}_{\mathrm{O}}$ content is only slightly lower (but statistically significant at $p<0.05)$ in $\mathrm{E}$ horizons $\left(0.82 \mathrm{~g} \mathrm{~kg}^{-1}\right)$ than in Eg horizons $\left(0.98 \mathrm{~g} \mathrm{~kg}^{-1}\right)$.

The soil material collected from the soil profiles $(<2 \mathrm{~mm})$ is composed mainly of $\mathrm{SiO}_{2}, \mathrm{Al}_{2} \mathrm{O}_{3}, \mathrm{Fe}_{2} \mathrm{O}_{3}$, and $\mathrm{K}_{2} \mathrm{O}$. The content of other compounds $\left(\mathrm{CaO}, \mathrm{MgO}, \mathrm{Na}_{2} \mathrm{O}, \mathrm{TiO}_{2}, \mathrm{P}_{2} \mathrm{O}_{5}\right.$, and $\mathrm{MnO}$ ) in almost all of the soils is below $1 \%$ (Table 4). Upper soil horizons (A, AE, E, and $\mathrm{Eg}$ ) contain a higher amount of $\mathrm{SiO}_{2}$ and a lower amount of $\mathrm{Al}_{2} \mathrm{O}_{3}, \mathrm{Fe}_{2} \mathrm{O}_{3}, \mathrm{~K}_{2} \mathrm{O}, \mathrm{CaO}$, and $\mathrm{MgO}$ in comparison with the lower part (Btx, Btg, BC, C) of the soils. However, slightly higher amount of $\mathrm{MgO}$ and $\mathrm{CaO}$ in surface horizons of some soils (e.g., Pleśna 2, Łazy 3, and Łazy 4) relative to the eluvial horizons is most likely caused by "biocycling." It should be noted that differences in the chemical composition of the $\mathrm{E}$ and Eg horizons are negligible (Table 4).

\subsection{Mineral composition of the eluvial horizons}

\subsubsection{X-ray diffraction}

The mineral composition of the clay fractions $(<2 \mu \mathrm{m})$ separated from the eluvial soil horizons showing strong, moderate, and very weak bleaching or lack of bleaching is very similar; thus, only the $\mathrm{x}$-ray patterns for selected horizons are presented (Fig. 2). The $x$-ray patterns show that the clay fraction is composed mainly of 2:1 clay minerals exhibiting a $\sim 1.4 \mathrm{~nm}$ peak in air dry state. The peak does not fully collapse to $1.0 \mathrm{~nm}$ neither after the heating of the samples at $330^{\circ} \mathrm{C}$ nor $550{ }^{\circ} \mathrm{C}$ (Fig. 2). However, it should be noted that the collapse of the $\sim 1.4$ to $1.0 \mathrm{~nm}$ peak is much stronger after the heating of specimens at $550{ }^{\circ} \mathrm{C}$. Treatment with glycerol also does not affect the $\sim 1.4 \mathrm{~nm}$ peak (Fig. 2). Kaolinite $(\sim 0.7 \mathrm{~nm}$ peak 
Table 3 Physical and chemical properties of the soils

\begin{tabular}{|c|c|c|c|c|c|c|c|c|c|c|c|}
\hline Horizon & Depth $(\mathrm{cm})$ & $\begin{array}{l}\text { Sand } \\
(\%)\end{array}$ & Silt & Clay & $\mathrm{Db}^{\mathrm{a}}\left(\mathrm{Mg} \mathrm{m}^{-3}\right)$ & $\mathrm{P}^{\mathrm{b}}(\%)$ & $\mathrm{pH} \mathrm{H} \mathrm{H}_{2} \mathrm{O}$ & Corg $(\%)$ & $\begin{array}{l}\mathrm{Fe}_{\mathrm{d}}^{\mathrm{c}} \\
\mathrm{g} \mathrm{kg}^{-1}\end{array}$ & $\mathrm{Fe}_{\mathrm{o}}{ }^{\mathrm{d}}$ & $\mathrm{Al}_{\mathrm{o}}{ }^{\mathrm{e}}$ \\
\hline
\end{tabular}

\begin{tabular}{|c|c|c|c|c|c|c|c|c|c|c|c|}
\hline \multicolumn{12}{|l|}{ Pleśna 1} \\
\hline $\mathrm{O}$ & $0-2$ & N.A. & N.A. & N.A. & N.A. & N.A. & N.A. & N.A. & N.A. & N.A. & N.A. \\
\hline A & $2-10$ & 17 & 68 & 15 & 1.18 & 53.7 & 4.2 & 1.2 & 4.91 & 2.6 & 0.9 \\
\hline $\mathrm{AE}$ & $10-30$ & 17 & 70 & 13 & 1.31 & 49.2 & 5.1 & 0.5 & 5.23 & 2.6 & 0.5 \\
\hline E & $30-70$ & 18 & 69 & 13 & 1.32 & 49.2 & 5.2 & 0.3 & 4.94 & 2.7 & 0.6 \\
\hline Btx & $70-105$ & 15 & 67 & 18 & 1.42 & 45.8 & 4.9 & 0.1 & 7.56 & 2.9 & 0.7 \\
\hline Btg & $105-150$ & 14 & 67 & 19 & 1.42 & 46.0 & 5.0 & 0.1 & 7.30 & 2.4 & 0.6 \\
\hline $\mathrm{BC}$ & $150-200$ & 21 & 66 & 13 & 1.40 & 47.2 & 5.3 & 0.1 & 6.57 & 1.5 & 0.3 \\
\hline \multicolumn{12}{|l|}{ Pleśna 2} \\
\hline A & $0-10$ & 13 & 71 & 16 & 1.50 & 42.3 & 5.0 & 0.7 & 5.65 & 3.2 & 0.8 \\
\hline $\mathrm{AE}$ & $10-30$ & 15 & 73 & 12 & 1.44 & 45.0 & 5.2 & 0.5 & 4.36 & 2.6 & 0.6 \\
\hline E & $30-55$ & 13 & 74 & 13 & 1.58 & 39.5 & 5.5 & 0.3 & 4.92 & 3.2 & 0.8 \\
\hline Btx1 & $55-80$ & 11 & 71 & 18 & 1.65 & 36.5 & 5.6 & 0.1 & 7.29 & 3.6 & 0.8 \\
\hline Btx2 & $80-160$ & 11 & 70 & 19 & 1.66 & 36.2 & 5.4 & 0.1 & 7.09 & 2.4 & 0.5 \\
\hline \multicolumn{12}{|l|}{ Pleśna 3} \\
\hline Ap & $0-35$ & 14 & 72 & 14 & 1.54 & 40.3 & 5.1 & 0.8 & 4.78 & 3.9 & 1.3 \\
\hline $\mathrm{Eg}$ & $35-45$ & 12 & 73 & 15 & 1.57 & 40.1 & 5.6 & 0.4 & 9.02 & 4.8 & 1.4 \\
\hline Btx1 & $45-100$ & 13 & 68 & 19 & 1.63 & 38.0 & 6.0 & 0.1 & 7.73 & 6.0 & 1.5 \\
\hline Btx2 & $100-180$ & 10 & 72 & 18 & 1.62 & 37.7 & 5.4 & 0.1 & 7.91 & 4.0 & 1.1 \\
\hline Btg & $180-245$ & 13 & 69 & 18 & 1.61 & 38.8 & 5.2 & 0.1 & 4.84 & 2.9 & 1.1 \\
\hline $\mathrm{BCg}$ & $245-285$ & 11 & 73 & 16 & N.A. & N.A. & 5.7 & 0.1 & 7.01 & 3.5 & 1.1 \\
\hline \multicolumn{12}{|l|}{ Pleśna 4} \\
\hline $\mathrm{O}$ & $0-2$ & N.A. & N.A. & N.A. & N.A. & N.A. & 4.3 & N.A. & N.A. & N.A. & N.A. \\
\hline A & $2-10$ & 21 & 66 & 13 & N.A. & N.A. & 4.1 & 3.8 & 4.46 & 2.9 & 0.9 \\
\hline E & $10-40$ & 13 & 74 & 13 & 1.26 & 52.6 & 4.2 & 0.4 & 4.22 & 2.9 & 0.8 \\
\hline $\mathrm{Eg}$ & $40-90$ & 13 & 74 & 13 & 1.58 & 40.8 & 4.3 & 0.1 & 4.23 & 3.2 & 0.7 \\
\hline Btx & $90-150$ & 9 & 66 & 25 & 1.63 & 39.6 & 5.2 & 0.1 & 6.65 & 2.6 & 0.6 \\
\hline \multicolumn{12}{|l|}{ Łazy 1} \\
\hline Ap & $0-35$ & 21 & 71 & 8 & 1.27 & 51.2 & 6.4 & 0.9 & 4.26 & 2.5 & 1.4 \\
\hline Eg & $35-50$ & 21 & 68 & 11 & 1.53 & 43.1 & 6.7 & 0.2 & 5.21 & 2.9 & 1.2 \\
\hline Btx & $50-70$ & 15 & 62 & 23 & 1.61 & 39.7 & 6.1 & 0.2 & 10.72 & 2.8 & 1.7 \\
\hline Btg & $70-90$ & 18 & 65 & 17 & 1.56 & 42.4 & 5.5 & 0.1 & 7.61 & 2.7 & 1.4 \\
\hline $\mathrm{BC}$ & $90-110$ & 16 & 71 & 13 & 1.56 & 41.8 & 5.7 & 0.1 & 5.92 & 2.9 & 1.1 \\
\hline $\mathrm{C}$ & $110-150$ & 18 & 69 & 13 & 1.65 & 38.7 & 5.6 & 0.1 & 4.35 & 2.7 & 1.0 \\
\hline \multicolumn{12}{|l|}{ Łazy 2} \\
\hline $\mathrm{Ol}$ & $0-5$ & N.A. & N.A. & N.A. & N.A. & N.A. & 4.2 & N.A. & N.A. & N.A. & N.A. \\
\hline Of & $5-8$ & N.A. & N.A. & N.A. & N.A. & N.A. & 4.5 & N.A. & N.A. & N.A. & N.A. \\
\hline A & $8-20$ & 23 & 64 & 13 & 0.80 & 68.3 & 3.9 & 3.0 & 5.67 & 3.2 & 0.9 \\
\hline E & $20-55$ & 11 & 78 & 11 & 1.30 & 50.8 & 4.2 & 0.4 & 5.05 & 2.6 & 0.8 \\
\hline Btx1 & $55-65$ & 18 & 63 & 19 & 1.44 & 46.3 & 4.0 & 0.3 & 8.33 & 2.5 & 1.2 \\
\hline Btx2 & $65-85$ & 18 & 63 & 19 & 1.60 & 39.8 & 4.7 & 0.2 & 8.94 & 1.8 & 1.0 \\
\hline $\mathrm{Bt}$ & $85-115$ & 20 & 64 & 16 & 1.60 & 40.7 & 5.0 & 0.2 & 7.33 & 1.8 & 0.6 \\
\hline $\mathrm{BC}$ & $115-135$ & 21 & 64 & 15 & 1.55 & 42.8 & 4.9 & 0.1 & 7.15 & 1.6 & 0.5 \\
\hline $\mathrm{C}$ & $135-185$ & 22 & 65 & 13 & 1.57 & 41.4 & 5.0 & 0.1 & 7.58 & 1.5 & 0.5 \\
\hline \multicolumn{12}{|l|}{ Łazy 3} \\
\hline $\mathrm{Ol}$ & $0-3$ & N.A. & N.A. & N.A. & N.A. & N.A. & N.A. & N.A. & N.A. & N.A. & N.A. \\
\hline A & $3-11$ & 18 & 70 & 12 & 1.19 & 54.6 & 6.6 & 1.1 & 5.54 & 2.5 & 0.9 \\
\hline $\mathrm{AE}$ & $11-25$ & 20 & 67 & 13 & 1.33 & 49.0 & 6.5 & 0.7 & 5.4 & 1.2 & 0.9 \\
\hline $\mathrm{Eg}$ & $25-50$ & 20 & 68 & 12 & 1.44 & 45.2 & 6.3 & 0.5 & 5.65 & 1.3 & 1.4 \\
\hline $\mathrm{Bh}$ & $50-70$ & 19 & 70 & 11 & 1.31 & 50.6 & 6.0 & 0.8 & 4.82 & 1.2 & 1.5 \\
\hline Btx1 & $70-110$ & 16 & 66 & 18 & 1.66 & 38.5 & 5.3 & 0.2 & 7.45 & 1.1 & 0.9 \\
\hline Btx2 & $110-160$ & 20 & 65 & 15 & N.A. & N.A. & 5.3 & 0.1 & 6.5 & 0.9 & 0.6 \\
\hline \multicolumn{12}{|l|}{ Łazy 4} \\
\hline $\mathrm{O}$ & $0-2$ & N.A. & N.A. & N.A. & N.A. & N.A. & N.A. & N.A. & N.A. & N.A. & N.A. \\
\hline $\mathrm{Ah}$ & $2-5$ & 21 & 68 & 11 & N.A. & N.A. & 4.3 & 6.1 & 5.61 & 1.2 & 0.9 \\
\hline $\mathrm{E}$ & $5-20$ & 19 & 69 & 12 & 1.40 & 47.2 & 4.4 & 0.6 & 4.48 & 1.1 & 0.7 \\
\hline $\mathrm{Eg}$ & $20-35$ & 21 & 68 & 11 & 1.48 & 42.6 & 4.6 & 0.4 & 4.31 & 1.1 & 0.5 \\
\hline Btx1 & $35-60$ & 16 & 70 & 14 & 1.55 & 41.5 & 5.5 & 0.2 & 5.96 & 1.1 & 0.6 \\
\hline Btx2 & $60-85$ & 14 & 63 & 23 & 1.59 & 41.5 & 5.3 & 0.1 & 10.3 & 0.9 & 0.6 \\
\hline Btg & $85-140$ & 19 & 64 & 17 & 1.56 & 41.4 & 5.4 & 0.1 & 7.05 & 0.8 & 0.5 \\
\hline $\mathrm{BCg}$ & $140-180$ & 19 & 67 & 14 & 1.58 & 39.9 & 5.3 & 0.1 & 6.22 & 0.7 & 0.4 \\
\hline \multicolumn{12}{|l|}{ Grabie } \\
\hline $\mathrm{Ol}$ & $0-2$ & N.A. & N.A. & N.A. & N.A. & N.A. & N.A. & N.A. & N.A. & N.A. & N.A. \\
\hline Ofh & $2-4$ & N.A. & N.A. & N.A. & N.A. & N.A. & 4.2 & N.A. & N.A. & N.A. & N.A. \\
\hline
\end{tabular}


Table 3 (continued)

\begin{tabular}{|c|c|c|c|c|c|c|c|c|c|c|c|}
\hline Horizon & Depth (cm) & $\begin{array}{l}\text { Sand } \\
(\%)\end{array}$ & Silt & Clay & $\mathrm{Db}^{\mathrm{a}}\left(\mathrm{Mg} \mathrm{m}^{-3}\right)$ & $\mathrm{P}^{\mathrm{b}}(\%)$ & $\mathrm{pH} \mathrm{H} \mathrm{H}_{2} \mathrm{O}$ & Corg $(\%)$ & $\begin{array}{l}\mathrm{Fe}_{\mathrm{d}}^{\mathrm{c}} \\
\mathrm{g} \mathrm{kg}^{-1}\end{array}$ & $\mathrm{Fe}_{\mathrm{o}}^{\mathrm{d}}$ & $\mathrm{Al}_{\mathrm{o}}^{\mathrm{e}}$ \\
\hline $\mathrm{Ah}$ & $4-12$ & 14 & 73 & 13 & N.A. & N.A. & 4.2 & 3.6 & 5.66 & 2.7 & 1.4 \\
\hline $\mathrm{Eg}$ & $12-35$ & 13 & 72 & 15 & 1.33 & 50.0 & 4.4 & 0.4 & 5.58 & 2.5 & 1.3 \\
\hline Btx1 & $35-70$ & 12 & 64 & 24 & 1.58 & 40.6 & 5.0 & 0.1 & 9.41 & 2.5 & 1.3 \\
\hline Btx2 & $70-120$ & 19 & 61 & 20 & 1.63 & 39.6 & 5.0 & 0.1 & 8.73 & 2.6 & 0.9 \\
\hline Btx3 & $120-155$ & 10 & 69 & 21 & 1.66 & 39.0 & 5.1 & 0.1 & 8.48 & 1.7 & 0.7 \\
\hline $2 \mathrm{C}$ & $155-165$ & 6 & 56 & 38 & N.A. & N.A. & 5.9 & 0.2 & N.A. & N.A. & N.A. \\
\hline \multicolumn{12}{|c|}{ Radziszów } \\
\hline Of & $0-2$ & N.A. & N.A. & N.A. & N.A. & N.A. & 5.0 & N.A. & N.A. & N.A. & N.A. \\
\hline $\mathrm{Ah}$ & $2-12$ & 18 & 69 & 13 & N.A. & N.A. & 4.3 & 3.6 & 5.4 & 3.1 & 1.4 \\
\hline $\mathrm{E}$ & $12-35$ & 13 & 77 & 10 & 1.21 & 54.0 & 4.5 & 0.3 & 5.27 & 2.9 & 1.3 \\
\hline $\mathrm{Eg}$ & $35-45$ & 12 & 79 & 9 & 1.41 & 47.2 & 4.5 & 0.2 & 4.62 & 2.2 & 0.9 \\
\hline Btx1 & $45-75$ & 11 & 68 & 21 & 1.57 & 41.9 & 5.0 & 0.1 & 8.59 & 2.3 & 1.3 \\
\hline Btx2 & $75-100$ & 12 & 70 & 18 & 1.63 & 40.3 & 5.2 & 0.1 & 8.63 & 2.7 & 1.1 \\
\hline Btx3 & $100-120$ & 15 & 53 & 32 & 1.78 & 35.0 & 5.5 & 0.1 & 13.31 & 2.2 & 1.0 \\
\hline $\mathrm{BC}$ & $120-130$ & 28 & 32 & 40 & 1.71 & 37.4 & 5.6 & 0.2 & 17.83 & 2.6 & 0.9 \\
\hline $2 \mathrm{C}$ & $130-160$ & 27 & 34 & 39 & N.A. & N.A. & 7.9 & 0.2 & N.A. & N.A. & N.A. \\
\hline \multicolumn{12}{|c|}{ Polanka Hallera } \\
\hline Ap & 0-20 & 13 & 74 & 13 & 1.40 & 46.7 & 6.9 & 1.2 & 5.3 & 1.1 & 0.5 \\
\hline A & $20-37$ & 11 & 76 & 11 & 1.53 & 39.4 & 7.3 & 0.8 & 5.57 & 1.2 & 0.7 \\
\hline $\mathrm{Eg}$ & $37-50$ & 12 & 79 & 8 & 1.60 & 38.5 & 7.5 & 0.2 & 5.61 & 1.0 & 0.4 \\
\hline Btx & $50-60$ & 12 & 69 & 19 & 1.65 & 35.7 & 7.5 & 0.1 & 8.44 & 1.0 & 0.8 \\
\hline Btg1 & $60-85$ & 10 & 70 & 19 & 1.65 & 43.1 & 7.4 & 0.1 & 8.69 & 0.9 & 0.7 \\
\hline Btg2 & $85-120$ & 12 & 69 & 19 & 1.67 & 33.9 & 5.6 & 0.1 & 6.51 & 0.7 & 0.4 \\
\hline Btg3 & $120-155$ & 10 & 74 & 16 & 1.73 & 35.0 & 6.0 & 0.1 & 5.13 & 0.8 & 0.4 \\
\hline Btg4 & $155-180$ & 10 & 70 & 19 & N.A. & N.A. & 6.7 & 0.1 & 10.98 & 1.0 & 0.4 \\
\hline \multicolumn{12}{|l|}{ Brzezie } \\
\hline Ap & $0-25$ & 14 & 71 & 15 & 1.55 & 40.8 & 5.3 & 1.1 & 5.32 & 3.7 & N.A. \\
\hline AE1 & $25-40$ & 10 & 75 & 15 & 1.50 & 43.8 & 5.4 & 0.7 & 5.65 & 4.2 & N.A. \\
\hline AE2 & $40-58$ & 9 & 76 & 15 & 1.45 & 45.3 & 5.4 & 0.7 & 5.62 & 4.3 & N.A. \\
\hline $\mathrm{Eg}$ & $58-70$ & 16 & 74 & 10 & 1.41 & 46.6 & 5.4 & 0.2 & 3.73 & 3.3 & N.A. \\
\hline Btx & $70-95$ & 11 & 67 & 22 & 1.68 & 37.5 & 5.1 & 0.1 & 8.50 & 3.8 & N.A. \\
\hline $\mathrm{Btg} 1$ & $95-115$ & 10 & 69 & 21 & 1.67 & 38.1 & 5.4 & 0.1 & 7.50 & 2.0 & N.A. \\
\hline Btg2 & 115-180 & 16 & 68 & 16 & 1.70 & 36.3 & 6.0 & 0.1 & 5.62 & 1.5 & N.A. \\
\hline
\end{tabular}

${ }^{\text {a }}$ Bulk density

${ }^{\mathrm{b}}$ Total porosity

${ }^{\mathrm{c}} \mathrm{CBD}$ extractable $\mathrm{Fe}$

${ }^{\mathrm{d}} \mathrm{AAO}$-extractable $\mathrm{Fe}$

${ }^{\text {e }}$ AAO-extractable Al

N.A. not analyzed

disappeared after heating at $\left.550{ }^{\circ} \mathrm{C}\right)$, mica $(\sim 1.0,0.5 \mathrm{~nm}$ peaks not affected by the various tests), and quartz ( $\sim 0.43 \mathrm{~nm}$ peak) are also present (Fig. 2). XRD results obtained for the clay fractions clearly indicate that the intensity of bleaching (strong vs. moderate or weak or lack), being a result of different duration and frequency of periodic water stagnation and redox processes above a hard and slowly permeable fragipan in the soils, does not affect the quality of clay minerals found in the Retisols of the Carpathian Foothills.

Quantitative mineral composition of the eluvial horizons (fraction $<2 \mathrm{~mm}$ ) is presented in Table 5, and selected $\mathrm{x}$-ray patterns presenting the bulk mineral composition are presented in Fig. 3. All of the eluvial horizons (E and Eg) are characterized by a predominance of quartz. Quartz content ranges from 60 to $72 \%$. The mean quartz content in the $\mathrm{E}$ and $\mathrm{Eg}$ horizons is 69 and $67 \%$, respectively. K-feldspar content ranges from 9 to $11 \%$ with a mean content of $10 \%$ in the $\mathrm{E}$ and Eg horizons. Plagioclase content is slightly lower than Kfeldspar content and ranges from 6 to $9 \%$. Mean plagioclase content in the $\mathrm{E}$ and $\mathrm{Eg}$ horizons is almost the same, i.e., 8 and $7 \%$, respectively. Dioctahedral mica found in the eluvial horizons ( $\mathrm{E}$ and $\mathrm{Eg}$ ) range between 3 and $5 \%$ with a mean content of $4 \%$ in the $\mathrm{E}$ and $\mathrm{Eg}$ horizons. Chlorite and kaolinite content in eluvial horizons is very low: 0-2\% chlorite, 1-2\% kaolinite. Mean chlorite content is $1 \%$ and mean kaolinite content is $2 \%$ in the $\mathrm{E}$ and $\mathrm{Eg}$ horizons of interest. The content of interlayered 2:1 clay minerals is between 4 and $8 \%$. The $\mathrm{E}$ and Eg horizons contain almost equal mean concentrations of the interlayered 2:1 clay minerals, i.e., 7 and $6 \%$, respectively. The results of both the qualitative analysis of clay fraction and the quantitative analysis of the bulk mineral composition of the eluvial horizons indicate a lack of significant differences at 
Table 4 Chemical composition of the soils

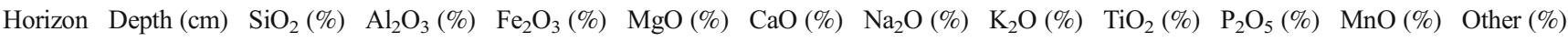

\begin{tabular}{|c|c|c|c|c|c|c|c|c|c|c|c|c|}
\hline \multicolumn{13}{|l|}{ Pleśna 1} \\
\hline $\mathrm{O}$ & $0-2$ & N.A. & N.A. & N.A. & N.A. & N.A. & N.A. & N.A. & N.A. & N.A. & N.A. & N.A. \\
\hline A & $2-10$ & 80.37 & 7.58 & 2.14 & 0.47 & 0.31 & 0.98 & 1.88 & 0.69 & 0.09 & 0.04 & 5.45 \\
\hline $\mathrm{AE}$ & $10-30$ & 81.34 & 7.81 & 2.17 & 0.50 & 0.40 & 1.01 & 1.98 & 0.73 & 0.09 & 0.06 & 3.91 \\
\hline $\mathrm{E}$ & $30-70$ & 82.35 & 7.49 & 1.96 & 0.47 & 0.37 & 0.97 & 1.91 & 0.71 & 0.08 & 0.07 & 3.62 \\
\hline Btx & $70-105$ & 77.94 & 9.40 & 3.18 & 0.74 & 0.44 & 0.94 & 2.13 & 0.71 & 0.08 & 0.06 & 4.38 \\
\hline Btg & $105-150$ & 78.47 & 9.25 & 3.09 & 0.73 & 0.45 & 0.93 & 2.16 & 0.68 & 0.10 & 0.06 & 4.08 \\
\hline $\mathrm{BC}$ & $150-200$ & 79.05 & 9.07 & 2.90 & 0.74 & 0.54 & 1.19 & 2.21 & 0.71 & 0.11 & 0.07 & 3.41 \\
\hline \multicolumn{13}{|c|}{ Pleśna 2} \\
\hline A & $0-10$ & 79.06 & 8.19 & 2.48 & 0.55 & 0.35 & 0.87 & 2.14 & 0.73 & 0.10 & 0.08 & 5.45 \\
\hline $\mathrm{AE}$ & $10-30$ & 82.67 & 7.35 & 1.90 & 0.43 & 0.35 & 0.91 & 2.06 & 0.76 & 0.10 & 0.09 & 3.38 \\
\hline $\mathrm{E}$ & $30-55$ & 81.47 & 7.80 & 1.99 & 0.46 & 0.38 & 0.90 & 2.14 & 0.77 & 0.09 & 0.12 & 3.88 \\
\hline Btx1 & $55-80$ & 77.95 & 9.48 & 3.11 & 0.73 & 0.42 & 0.83 & 2.29 & 0.73 & 0.08 & 0.06 & 4.32 \\
\hline Btx2 & $80-160$ & 78.38 & 9.48 & 3.07 & 0.73 & 0.44 & 0.93 & 2.32 & 0.72 & 0.09 & 0.07 & 3.77 \\
\hline \multicolumn{13}{|l|}{ Pleśna 3} \\
\hline Ap & $0-35$ & 80.62 & 7.68 & 2.24 & 0.47 & 0.40 & 0.87 & 2.07 & 0.76 & 0.16 & 0.10 & 4.63 \\
\hline Btx1 & $45-100$ & 77.35 & 9.52 & 3.27 & 0.73 & 0.51 & 0.83 & 2.32 & 0.73 & 0.11 & 0.07 & 4.56 \\
\hline Btx2 & $100-180$ & 78.07 & 9.43 & 3.15 & 0.74 & 0.50 & 0.92 & 2.38 & 0.72 & 0.12 & 0.05 & 3.92 \\
\hline Btg & $180-245$ & 79.34 & 9.14 & 2.60 & 0.70 & 0.51 & 0.95 & 2.29 & 0.72 & 0.10 & 0.06 & 3.59 \\
\hline $\mathrm{BCg}$ & $245-285$ & 78.72 & 9.35 & 3.00 & 0.72 & 0.55 & 1.01 & 2.36 & 0.73 & 0.12 & 0.07 & 3.37 \\
\hline \multicolumn{13}{|l|}{ Pleśna 4} \\
\hline $\mathrm{O}$ & $0-2$ & N.A. & N.A. & N.A. & N.A. & N.A. & N.A. & N.A. & N.A. & N.A. & N.A. & N.A. \\
\hline A & $2-10$ & 77.38 & 6.61 & 1.57 & 0.32 & 0.30 & 0.88 & 1.83 & 0.69 & 0.10 & 0.02 & 10.30 \\
\hline $\mathrm{E}$ & $10-40$ & 82.73 & 7.37 & 1.81 & 0.40 & 0.31 & 0.98 & 2.07 & 0.76 & 0.05 & 0.06 & 3.46 \\
\hline $\mathrm{Eg}$ & $40-90$ & 82.91 & 7.60 & 1.88 & 0.43 & 0.33 & 0.99 & 2.11 & 0.76 & 0.10 & 0.09 & 2.80 \\
\hline Btx & $90-150$ & 77.57 & 9.60 & 3.26 & 0.77 & 0.49 & 0.93 & 2.32 & 0.72 & 0.10 & 0.06 & 4.18 \\
\hline \multicolumn{13}{|l|}{ Łazy 1} \\
\hline Ap & $0-35$ & 83.12 & 6.38 & 1.67 & 0.38 & 0.55 & 0.85 & 1.93 & 0.66 & 0.20 & 0.07 & 4.19 \\
\hline $\mathrm{Eg}$ & $35-50$ & 83.49 & 7.15 & 2.00 & 0.46 & 0.44 & 0.92 & 2.03 & 0.68 & 0.07 & 0.06 & 2.70 \\
\hline Btx & $50-70$ & 79.34 & 8.75 & 3.09 & 0.68 & 0.48 & 0.83 & 2.08 & 0.65 & 0.07 & 0.05 & 3.98 \\
\hline Btg & $70-90$ & 79.85 & 8.51 & 3.17 & 0.64 & 0.43 & 0.84 & 2.06 & 0.66 & 0.07 & 0.05 & 3.72 \\
\hline $\mathrm{BC}$ & $90-110$ & 81.35 & 7.93 & 2.63 & 0.58 & 0.43 & 0.82 & 1.99 & 0.65 & 0.08 & 0.05 & 3.49 \\
\hline $\mathrm{Ol}$ & $0-5$ & N.A. & N.A. & N.A. & N.A. & N.A. & N.A. & N.A. & N.A. & N.A. & N.A. & N.A. \\
\hline Of & $5-8$ & N.A. & N.A. & N.A. & N.A. & N.A. & N.A. & N.A. & N.A. & N.A. & N.A. & N.A. \\
\hline A & $8-20$ & N.A. & N.A. & N.A. & N.A. & N.A. & N.A. & N.A. & N.A. & N.A. & N.A. & N.A. \\
\hline $\mathrm{E}$ & $20-55$ & 83.75 & 6.12 & 1.70 & 0.34 & 0.25 & 0.79 & 1.71 & 0.64 & 0.08 & 0.04 & 4.58 \\
\hline Btx1 & $55-65$ & 80.41 & 8.04 & 2.77 & 0.59 & 0.23 & 0.76 & 1.87 & 0.64 & 0.08 & 0.05 & 4.56 \\
\hline Btx2 & $65-85$ & 79.20 & 8.70 & 3.20 & 0.68 & 0.35 & 0.78 & 1.92 & 0.64 & 0.10 & 0.05 & 4.38 \\
\hline $\mathrm{Bt}$ & $85-115$ & 80.66 & 8.18 & 2.78 & 0.63 & 0.39 & 0.81 & 1.92 & 0.63 & 0.09 & 0.05 & 3.86 \\
\hline $\mathrm{BC}$ & $115-135$ & 82.16 & 7.66 & 2.49 & 0.56 & 0.40 & 0.83 & 1.86 & 0.62 & 0.07 & 0.05 & 3.30 \\
\hline $\mathrm{C}$ & $135-185$ & 82.01 & 7.54 & 2.61 & 0.55 & 0.40 & 0.86 & 1.90 & 0.60 & 0.08 & 0.06 & 3.39 \\
\hline \multicolumn{13}{|l|}{ Łazy 3} \\
\hline $\mathrm{Ol}$ & $0-3$ & N.A. & N.A. & N.A. & N.A. & N.A. & N.A. & N.A. & N.A. & N.A. & N.A. & N.A. \\
\hline A & $3-11$ & 81.56 & 6.79 & 1.87 & 0.43 & 0.51 & 0.79 & 1.88 & 0.66 & 0.18 & 0.07 & 5.26 \\
\hline $\mathrm{AE}$ & $11-25$ & 83.50 & 6.53 & 1.74 & 0.39 & 0.44 & 0.78 & 1.81 & 0.67 & 0.14 & 0.08 & 3.92 \\
\hline $\mathrm{Eg}$ & $25-50$ & 82.89 & 6.94 & 1.79 & 0.38 & 0.40 & 0.79 & 1.96 & 0.69 & 0.16 & 0.11 & 3.89 \\
\hline $\mathrm{Bh}$ & $50-70$ & 83.43 & 6.72 & 1.61 & 0.38 & 0.37 & 0.81 & 1.98 & 0.67 & 0.12 & 0.07 & 3.84 \\
\hline Btx1 & $70-110$ & 80.18 & 8.28 & 2.83 & 0.63 & 0.37 & 0.73 & 1.98 & 0.66 & 0.11 & 0.05 & 4.18 \\
\hline Btx2 & $110-160$ & 82.09 & 7.54 & 2.48 & 0.57 & 0.37 & 0.80 & 1.93 & 0.62 & 0.07 & 0.04 & 3.49 \\
\hline \multicolumn{13}{|l|}{ Łazy 4} \\
\hline $\mathrm{Ol}$ & $0-2$ & N.A. & N.A. & N.A. & N.A. & N.A. & N.A. & N.A. & N.A. & N.A. & N.A. & N.A. \\
\hline $\mathrm{Ah}$ & $2-5$ & 71.41 & 6.06 & 2.05 & 0.35 & 0.39 & 0.72 & 1.63 & 0.62 & 0.15 & 0.04 & 16.58 \\
\hline $\mathrm{E}$ & $5-20$ & 84.19 & 6.45 & 1.53 & 0.35 & 0.29 & 0.89 & 1.94 & 0.68 & 0.08 & 0.05 & 3.55 \\
\hline $\mathrm{Eg}$ & $20-35$ & 84.59 & 6.48 & 1.51 & 0.34 & 0.29 & 0.89 & 1.95 & 0.70 & 0.08 & 0.07 & 3.10 \\
\hline Btx1 & $35-60$ & 82.21 & 7.76 & 2.23 & 0.55 & 0.36 & 0.94 & 2.17 & 0.72 & 0.09 & 0.06 & 2.91 \\
\hline Btx2 & $60-85$ & 77.66 & 9.21 & 3.65 & 0.76 & 0.41 & 0.81 & 2.11 & 0.69 & 0.06 & 0.05 & 4.59 \\
\hline Btg & $85-140$ & 80.51 & 8.44 & 2.77 & 0.64 & 0.40 & 0.90 & 2.07 & 0.65 & 0.07 & 0.06 & 3.49 \\
\hline $\mathrm{BCg}$ & $140-180$ & 81.57 & 7.96 & 2.41 & 0.58 & 0.41 & 0.94 & 2.14 & 0.67 & 0.06 & 0.06 & 3.20 \\
\hline \multicolumn{13}{|l|}{ Grabie } \\
\hline $\mathrm{Ol}$ & $0-2$ & N.A. & N.A. & N.A. & N.A. & N.A. & N.A. & N.A. & N.A. & N.A. & N.A. & N.A. \\
\hline Ofh & $2-4$ & N.A. & N.A. & N.A. & N.A. & N.A. & N.A. & N.A. & N.A. & N.A. & N.A. & N.A. \\
\hline $\mathrm{Ah}$ & $4-12$ & 77.57 & 6.35 & 1.73 & 0.34 & 0.29 & 0.68 & 1.87 & 0.66 & 0.11 & 0.03 & 10.37 \\
\hline
\end{tabular}


Table 4 (continued)

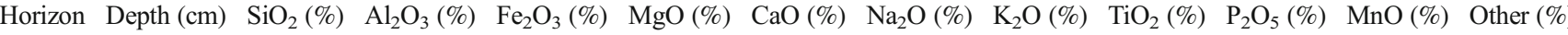

\begin{tabular}{|c|c|c|c|c|c|c|c|c|c|c|c|c|}
\hline $\mathrm{Eg}$ & $12-35$ & 82.20 & 7.76 & 2.06 & 0.46 & 0.33 & 0.82 & 2.34 & 0.75 & 0.12 & 0.07 & 3.09 \\
\hline Btx1 & $35-70$ & 77.19 & 9.66 & 3.43 & 0.68 & 0.37 & 0.77 & 2.32 & 0.72 & 0.12 & 0.04 & 4.70 \\
\hline Btx2 & $70-120$ & 76.81 & 9.78 & 3.33 & 0.73 & 0.46 & 0.87 & 2.44 & 0.73 & 0.10 & 0.05 & 4.70 \\
\hline Btx3 & $120-155$ & 76.05 & 10.36 & 3.42 & 0.86 & 0.58 & 1.04 & 2.53 & 0.76 & 0.10 & 0.06 & 4.24 \\
\hline $2 \mathrm{C}$ & $155-165$ & 62.95 & 15.59 & 5.86 & 2.31 & 0.77 & 1.21 & 3.10 & 0.73 & 0.16 & 0.11 & 7.21 \\
\hline \multicolumn{13}{|c|}{ Radziszów } \\
\hline Of & $0-2$ & N.A. & N.A. & N.A. & N.A. & N.A. & N.A. & N.A. & N.A. & N.A. & N.A. & N.A. \\
\hline $\mathrm{Ah}$ & $2-12$ & 77.50 & 6.55 & 1.67 & 0.32 & 0.33 & 0.73 & 1.98 & 0.69 & 0.08 & 0.03 & 10.12 \\
\hline $\mathrm{E}$ & $12-35$ & 83.23 & 7.22 & 1.73 & 0.37 & 0.31 & 0.79 & 2.17 & 0.74 & 0.05 & 0.05 & 3.34 \\
\hline $\mathrm{Eg}$ & $35-45$ & 84.13 & 7.06 & 1.63 & 0.38 & 0.33 & 0.85 & 2.28 & 0.74 & 0.04 & 0.04 & 2.52 \\
\hline Btx1 & $45-75$ & 78.32 & 9.47 & 3.18 & 0.64 & 0.35 & 0.78 & 2.34 & 0.72 & 0.06 & 0.04 & 4.10 \\
\hline Btx2 & $75-100$ & 76.97 & 9.84 & 3.44 & 0.69 & 0.44 & 0.86 & 2.33 & 0.75 & 0.07 & 0.04 & 4.57 \\
\hline Btx3 & $100-120$ & 71.86 & 11.92 & 4.53 & 1.04 & 0.59 & 0.71 & 2.52 & 0.75 & 0.07 & 0.05 & 5.96 \\
\hline $\mathrm{BC}$ & $120-130$ & 67.23 & 12.98 & 5.61 & 1.34 & 0.68 & 0.50 & 2.58 & 0.68 & 0.10 & 0.08 & 8.22 \\
\hline $2 \mathrm{C}$ & $130-160$ & 65.62 & 12.37 & 4.77 & 1.32 & 3.10 & 0.45 & 2.56 & 0.63 & 0.23 & 0.05 & 8.90 \\
\hline \multicolumn{13}{|c|}{ Polanka Hallera } \\
\hline Ap & $0-20$ & 79.73 & 6.93 & 2.07 & 0.39 & 0.71 & 0.78 & 2.07 & 0.71 & 0.19 & 0.07 & 6.36 \\
\hline A & $20-37$ & 80.69 & 6.96 & 2.05 & 0.39 & 0.71 & 0.80 & 2.07 & 0.73 & 0.15 & 0.07 & 5.40 \\
\hline $\mathrm{Eg}$ & $37-50$ & 84.16 & 7.23 & 1.94 & 0.42 & 0.49 & 0.89 & 2.29 & 0.77 & 0.08 & 0.04 & 1.70 \\
\hline Btx & $50-60$ & 79.04 & 8.98 & 3.09 & 0.61 & 0.57 & 0.80 & 2.29 & 0.77 & 0.08 & 0.08 & 3.70 \\
\hline Btg1 & $60-85$ & 77.86 & 9.33 & 3.25 & 0.50 & 0.56 & 0.83 & 2.29 & 0.75 & 0.05 & 0.05 & 4.54 \\
\hline Btg2 & $85-120$ & 79.00 & 8.57 & 3.21 & 0.57 & 0.51 & 0.87 & 2.23 & 0.72 & 0.06 & 0.07 & 4.24 \\
\hline Btg3 & $120-155$ & 79.73 & 9.24 & 2.74 & 0.64 & 0.55 & 0.98 & 2.38 & 0.75 & 0.06 & 0.05 & 2.90 \\
\hline Btg4 & $155-180$ & N.A. & N.A. & N.A. & N.A. & N.A. & N.A. & N.A. & N.A. & N.A. & N.A. & N.A. \\
\hline \multicolumn{13}{|l|}{ Brzezie } \\
\hline Ap & $0-25$ & 80.00 & 7.65 & 2.23 & 0.46 & 0.47 & 0.79 & 1.99 & 0.66 & 0.15 & 0.06 & 5.54 \\
\hline AE1 & $25-40$ & 80.22 & 7.82 & 2.28 & 0.47 & 0.46 & 0.78 & 2.02 & 0.68 & 0.13 & 0.06 & 5.08 \\
\hline AE2 & $40-58$ & 80.65 & 7.81 & 2.16 & 0.46 & 0.43 & 0.76 & 2.01 & 0.68 & 0.12 & 0.05 & 4.87 \\
\hline $\mathrm{Eg}$ & $58-70$ & 84.77 & 6.68 & 1.53 & 0.36 & 0.35 & 0.79 & 2.03 & 0.67 & 0.06 & 0.07 & 2.69 \\
\hline Btx & $70-95$ & 78.63 & 9.05 & 3.15 & 0.64 & 0.42 & 0.74 & 2.14 & 0.69 & 0.06 & 0.05 & 4.43 \\
\hline Btg1 & $95-115$ & 79.91 & 8.62 & 2.91 & 0.60 & 0.45 & 0.80 & 2.14 & 0.66 & 0.05 & 0.04 & 3.82 \\
\hline Btg2 & $115-180$ & 81.05 & 8.10 & 2.46 & 0.55 & 0.53 & 0.90 & 2.16 & 0.67 & 0.07 & 0.04 & 3.47 \\
\hline
\end{tabular}

${ }^{\text {a }}$ Loss on ignition

N.A. not analyzed

$p<0.05$ ( $t$ Student test) between $\mathrm{E}$ and $\mathrm{Eg}$ horizons in the Retisols. This is most likely due to the fact that the Retisols of the Carpathian Foothills contain very little iron-bearing minerals.

\subsubsection{FTIR spectroscopy}

Analysis of FTIR spectra obtained for the clay fractions $(<2 \mu \mathrm{m})$ separated from eluvial horizons supports the earlier interpretation of XRD data. Due to the fact that all of the obtained FTIR spectra are almost identical, only spectra for selected eluvial horizons are presented (Fig. 4). Assignments for the major FTIR bands are presented in Table 6. Two strong absorption bands at 3699 and $\sim 3620 \mathrm{~cm}^{-1}$ are present in the $\mathrm{OH}$ stretching region and indicate presence of kaolinite and dioctahedral 2:1 clay minerals (Table 6). All weak absorption bands in the $\mathrm{OH}$ stretching region are related to vibrations of $\mathrm{Al}-\mathrm{OH}-\mathrm{Al}$ in the structure of kaolinite and/or dioctahedral mica (Table 6, Fig. 5) (Besson and Drits 1997; Madejová
2003; Zviagina et al. 2004). Strong absorption bands present at $1165,1085,799$, and $780 \mathrm{~cm}^{-1}$ in the $\mathrm{Si}-\mathrm{O}$ and $\mathrm{OH}$ bending regions are related to quartz and silicates (Table 6, Fig. 4). In addition, two broad bands at $\sim 1620$ and $\sim 1405 \mathrm{~cm}^{-1}$ are present and originate from organic matter (Table 6) (Celi et al. 1997; Cocozza et al. 2003; Tivet et al. 2013) and water, because the bands become weaker after heating at $180{ }^{\circ} \mathrm{C}$ and disappear almost completely after treatment with hydrogen peroxide (see Szymański et al. 2014). The presence of 2926 and $\sim 2855 \mathrm{~cm}^{-1}$ bands is associated with methyl and methylene groups, respectively (Ellerbrock and Gerke 2004), while the wide band at $23430 \mathrm{~cm}^{-1}$ indicates $\mathrm{H}-\mathrm{O}-\mathrm{H}$ vibrations of structurally adsorbed water molecules (Table 6) (Bain and Fraser 1994; Besson and Drits 1997; Madejová 2003). The FTIR data also indicate the lack of discernible water stagnation effects and redox effects on the different transformation pathways of clay minerals in strongly, moderately, and weakly bleached eluvial horizons as well as in eluvial horizons without bleaching. 

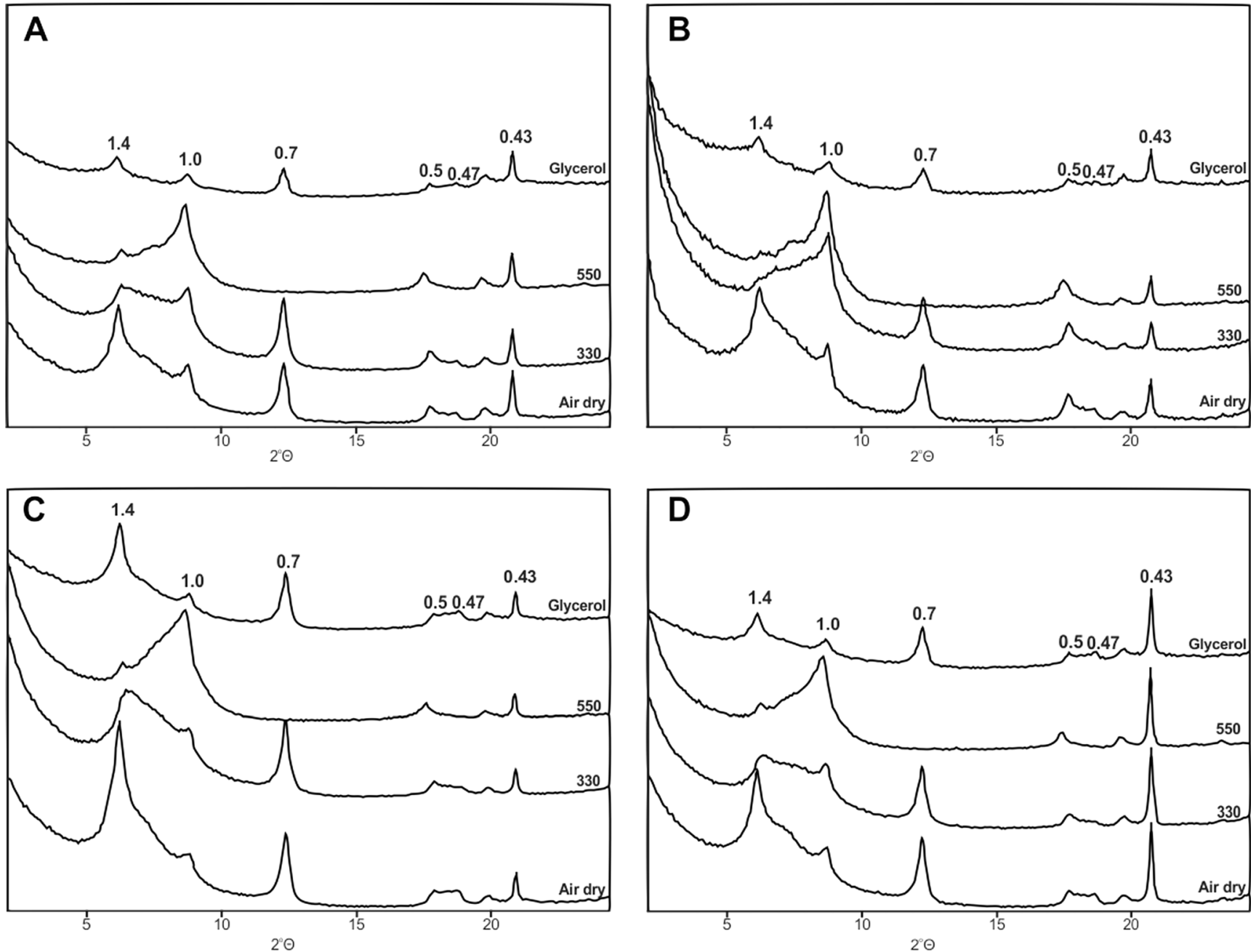

Fig. 2 XRD patterns of the clay fraction $(<2 \mu \mathrm{m})$ from selected eluvial horizons: Eg horizon of the Brzezie profile (a); E horizon of the Pleśna 1 profile (b); E horizon of the Radziszów profile (c); Eg horizon of the Radziszów profile (d)

Table 5 Quantitative mineral composition of bulk samples $(<2 \mathrm{~mm})$ of the $\mathrm{E}$ and Eg horizons

\begin{tabular}{|c|c|c|c|c|c|c|c|c|}
\hline Profile and horizon & Quartz (\%) & $\begin{array}{l}\mathrm{K}- \\
\text { feldspars }(\%)\end{array}$ & Plagioclases (\%) & $\begin{array}{l}\text { Dioctahedral } \\
\text { micas }(\%)\end{array}$ & $\begin{array}{l}\text { Chlorite } \\
(\%)\end{array}$ & Kaolinite (\%) & $\begin{array}{l}\text { Interlayered } 2: 1 \\
\text { clay minerals }(\%)\end{array}$ & Amorphous (\%) \\
\hline Pleśna 1 E & 68 & 9 & 9 & 4 & 1 & 2 & 6 & 1 \\
\hline Pleśna 2 E & 67 & 10 & 8 & 4 & 2 & 1 & 7 & 1 \\
\hline Pleśna 3 Eg & 60 & 9 & 8 & 4 & 1 & 2 & 6 & 10 \\
\hline Pleśna 4 E & 70 & 10 & 9 & 3 & 0 & 1 & 6 & 1 \\
\hline Pleśna 4 Eg & 69 & 10 & 9 & 4 & 0 & 2 & 5 & 1 \\
\hline Łazy $1 \mathrm{Eg}$ & 63 & 9 & 7 & 4 & 1 & 2 & 7 & 7 \\
\hline Łazy 2 E & 69 & 9 & 7 & 4 & 2 & 2 & 7 & 0 \\
\hline Łazy $3 \mathrm{Eg}$ & 69 & 9 & 6 & 5 & 0 & 2 & 6 & 3 \\
\hline Łazy 4 E & 70 & 10 & 8 & 3 & 1 & 1 & 7 & 0 \\
\hline Łazy 4 Eg & 71 & 10 & 8 & 4 & 0 & 2 & 5 & 0 \\
\hline Grabie Eg & 64 & 11 & 7 & 5 & 2 & 1 & 6 & 4 \\
\hline Radziszów E & 69 & 11 & 7 & 3 & 0 & 2 & 8 & 0 \\
\hline Radziszów Eg & 71 & 10 & 7 & 4 & 2 & 2 & 4 & 0 \\
\hline $\begin{array}{l}\text { Polanka Hallera } \\
\text { Eg }\end{array}$ & 67 & 11 & 8 & 4 & 1 & 2 & 6 & 1 \\
\hline Brzezie Eg & 68 & 9 & 7 & 3 & 1 & 1 & 6 & 5 \\
\hline
\end{tabular}


Fig. 3 XRD patterns of bulk samples $(<2 \mathrm{~mm})$ of the selected eluvial horizons from: Eg horizon of the Polanka Hallera profile (a); Eg horizon of the Radziszów profile (b); E horizon of the Radziszów profile (c); E horizon of the Pleśna 1 profile (d)

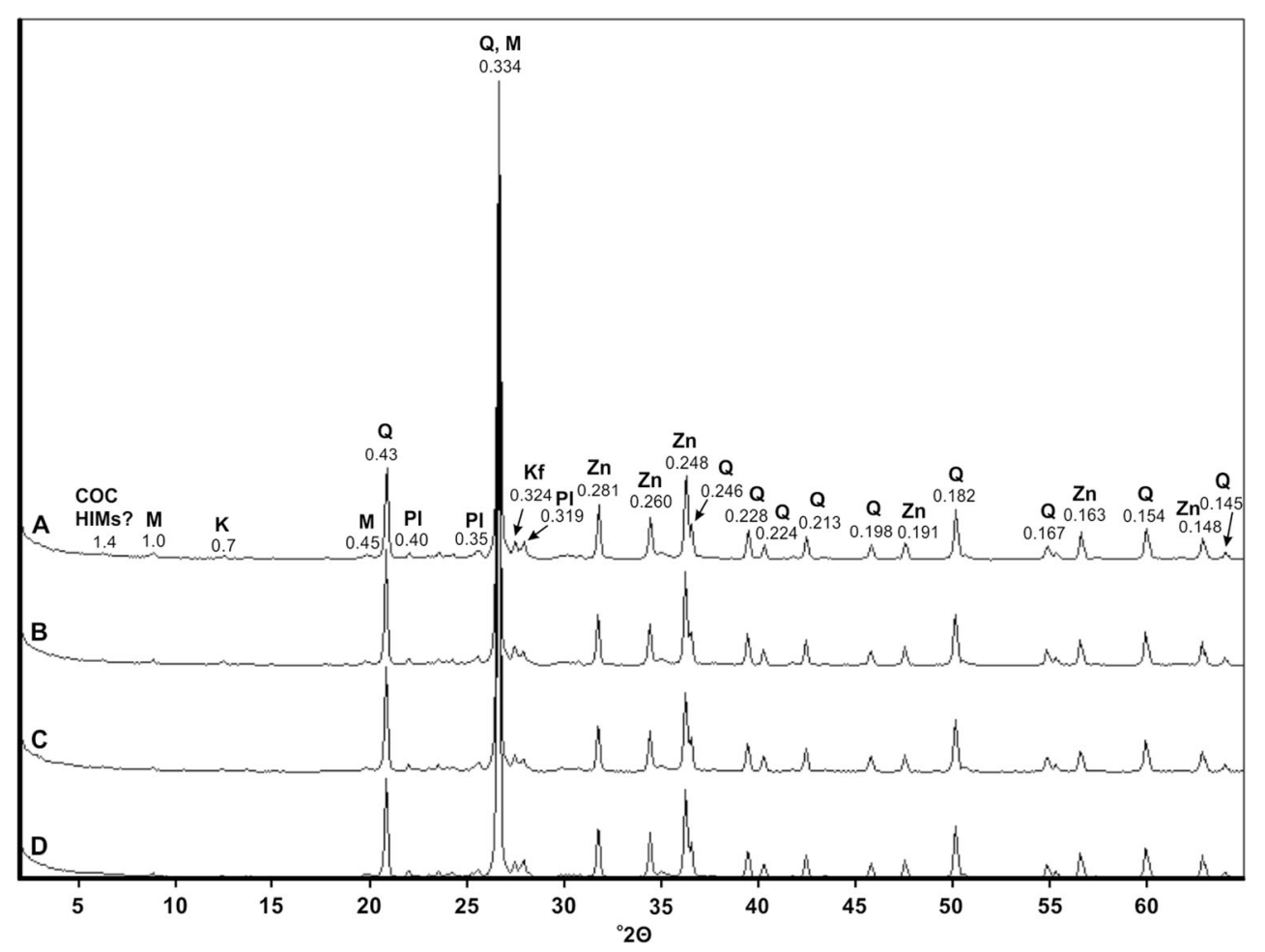

\subsubsection{Mössbauer spectroscopy}

Mössbauer spectroscopy results obtained for the clay fractions are shown in Table 7, and selected Mössbauer spectra are shown in Fig. 6. The data indicate that the relative content of $\mathrm{Fe}^{3+}$ and $\mathrm{Fe}^{2+}$ is not related to the intensity of bleaching of eluvial horizons within the same soil profile and between different profiles. In case of Lazy 4 and Radziszów profiles, relative $\mathrm{Fe}^{2+}$ content is higher in the Eg horizons than in the E horizons while in Pleśna 4 profile, the relative content of $\mathrm{Fe}^{2+}$ is lower in the Eg horizon than in the $\mathrm{E}$ horizon. The same is true for $\mathrm{E}$ and $\mathrm{Eg}$ horizons originating from different soil profiles - the relative content of $\mathrm{Fe}^{2+}$ is higher in the $\mathrm{Eg}$ horizons from the Pleśna 3 profile than in the E horizon from the Pleśna 1 profile, but at the same time, it is lower than the relative content of $\mathrm{Fe}^{2+}$ in the $\mathrm{E}$ horizon from the Pleśna 4
Fig. 4 FTIR spectra of the clay fraction $(<2 \mu \mathrm{m})$ from selected eluvial horizons: Eg horizon of the Brzezie profile (a); E horizon of the Pleśna 1 profile (b); E horizon of the Radziszów profile (c); Eg horizon of the Radziszów profile (d)

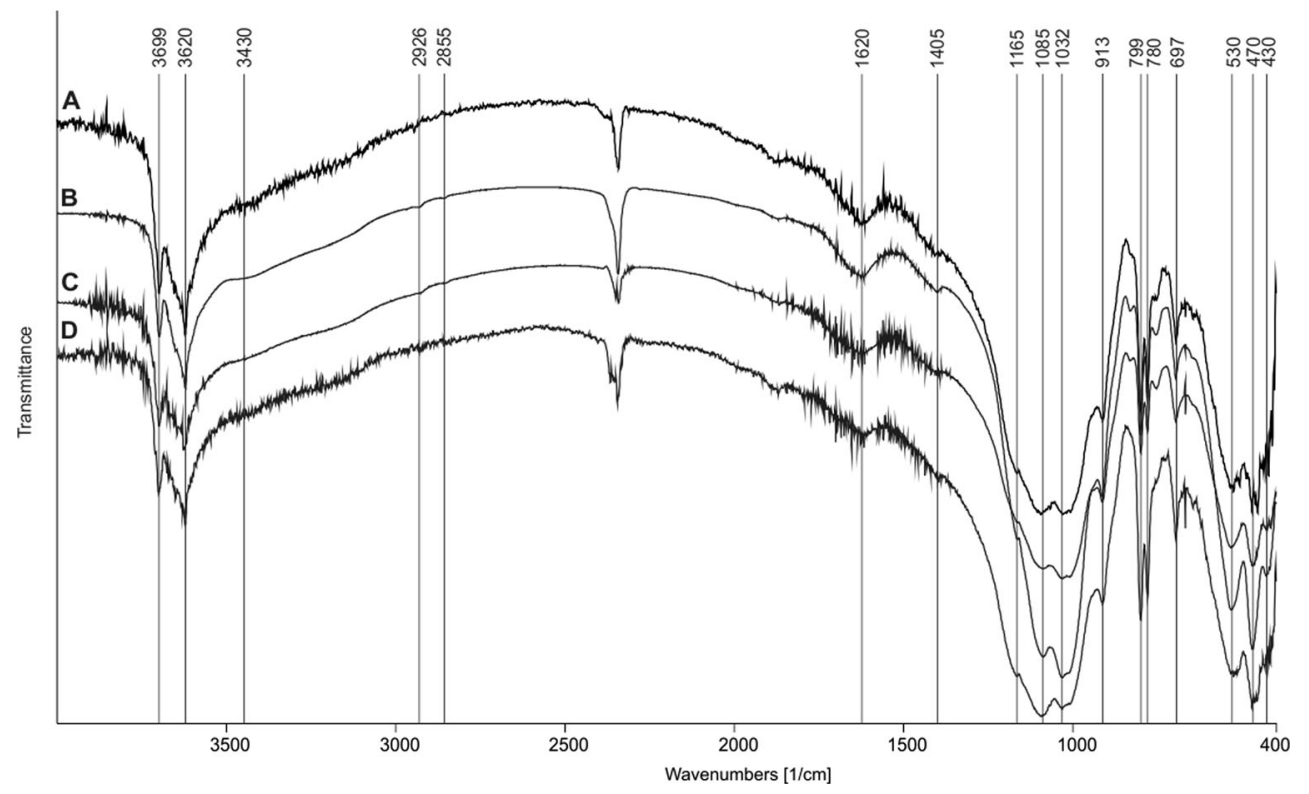


Table 6 Assignments for the major FTIR bands

\begin{tabular}{ll}
\hline FTIR peak position & Assignment \\
\hline $\mathrm{cm}^{-1}$ & \\
3699 & $\mathrm{Al}-\mathrm{OH}-\mathrm{Al}$ of kaolinite \\
3620 & $\mathrm{~A}-\mathrm{OH}-\mathrm{Al}$ of kaolinite and/or dioctahedral $2: 1$ clay minerals \\
3655 & $\mathrm{Al}-\mathrm{OH}-\mathrm{Al}$ of kaolinite and/or dioctahedral $2: 1$ clay minerals \\
3687 & $\mathrm{Al}-\mathrm{OH}-\mathrm{Al}$ of kaolinite and/or dioctahedral $2: 1$ clay minerals \\
3674 & $\mathrm{Al}-\mathrm{OH}-\mathrm{Al}$ of kaolinite and/or dioctahedral $2: 1$ clay minerals \\
3646 & $\mathrm{Al}-\mathrm{OH}-\mathrm{Al}$ of kaolinite and/or dioctahedral $2: 1$ clay minerals \\
3633 & $\mathrm{Al}-\mathrm{OH}-\mathrm{Al}$ of kaolinite and/or dioctahedral $2: 1$ clay minerals \\
3626 & $\mathrm{Al}-\mathrm{OH}-\mathrm{Al}$ of kaolinite and/or dioctahedral 2:1 clay minerals \\
3430 & $\mathrm{H}-\mathrm{O}-\mathrm{H}$ of water molecules \\
2926 & $\mathrm{C}-\mathrm{H}$ of methyl groups \\
2855 & $\mathrm{C}-\mathrm{H}$ of methylene groups \\
1620 & aromatic C=C and C=O of carboxylic acid \\
1405 & aliphatic C-H and C-OH deformation of COOH groups \\
1165 & Si-O stretching of quartz and silicates \\
1085 & Si-O stretching of quartz and silicates \\
799 & Si-O stretching of quartz and silicates \\
780 & Si-O stretching of quartz and silicates \\
&
\end{tabular}

profile (Table 7). The mean relative content of $\mathrm{Fe}^{2+}$ in the clay fraction in the Eg horizons is only slightly higher (7.7 \%) than the mean for the clay fraction obtained from $\mathrm{E}$ horizons $(7.6 \%)$. These results also indicate a lack of relationship between water stagnation and redox processes and relative content of iron in different oxidation states in the clay fraction of the eluvial horizons (E and $\mathrm{Eg}$ ).

\subsection{Verification of ferrolysis occurrence in Retisols}

The Retisols with Eg horizons showing strong bleaching (Pleśna 3, Pleśna 4, Łazy 1, Polanka Hallera, and Brzezie profiles) are very similar to the ferrolyzed soils studied by Brinkman (1979). They are characterized by a strongly bleached, coarser-grained horizon underlain by mottled, finer-grained horizons with bleached vertical tongues (Szymański et al. 2011). In addition, the bleached horizons contain a large number of $\mathrm{Fe}-\mathrm{Mn}$ nodules indicating redox processes, and all of the soils are characterized by acidic or slightly acidic $\mathrm{pH}$, with the exception of the Polanka Hallera profile. The strongly bleached Eg horizons of the Retisols exhibit the occurrence of clay minerals with interlayering characterized by a lack of complete collapse after heating as well as a lack of discernible swelling after glycerol solvation (Fig. 2). All the above-mentioned characteristics may indicate that ferrolysis occurs in these soils. However, the same clay minerals featuring interlayering were also identified in other Eg horizons with moderate or very weak bleaching as well as in E horizons, which do not exhibit bleaching at all and contain either few or no $\mathrm{Fe}-\mathrm{Mn}$ nodules indicating very weak or a lack of redox processes. Thus, the obtained research results indicate the lack of a clear influence of redox processes (due to

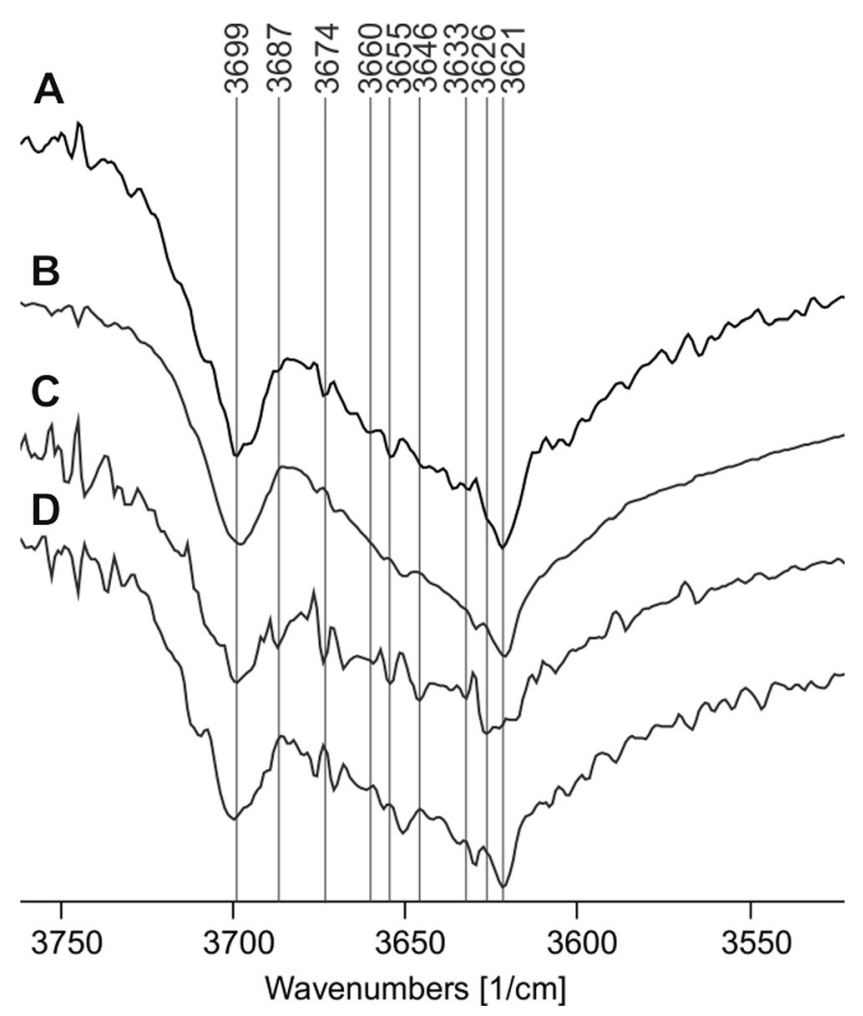

Fig. 5 FTIR spectra of the clay fraction $(<2 \mu \mathrm{m})$ in the $\mathrm{OH}$ stretching region from selected eluvial horizons: Eg horizon of the Brzezie profile (a); E horizon of the Pleśna 1 profile (b); E horizon of the Radziszów profile (c); Eg horizon of the Radziszów profile (d) 
Table 7 Relative content of $\mathrm{Fe}^{2+}$ and $\mathrm{Fe}^{3+}$ in crystalline structure of clay fraction $(<0.002 \mathrm{~mm})$ in the $\mathrm{E}$ and $\mathrm{Eg}$ horizons

\begin{tabular}{|c|c|c|c|c|}
\hline Horizon & Iron valence state & $\begin{array}{l}\mathrm{A}^{\mathrm{a}}(\%) \\
\pm 0.2\end{array}$ & $\begin{array}{l}\mathrm{IS}^{\mathrm{b}}(\mathrm{mm} / \mathrm{s}) \\
\pm 0.01\end{array}$ & $\begin{array}{l}\mathrm{QS}^{\mathrm{c}}(\mathrm{mm} / \mathrm{s}) \\
\pm 0.01\end{array}$ \\
\hline
\end{tabular}

\begin{tabular}{clrrr}
\hline Pleśna 1 & & & & \\
$\mathrm{E}$ & $\mathrm{Fe}^{3+}$ & 95.0 & 0.38 & 0.65 \\
& $\mathrm{Fe}^{2+}$ & 5.0 & 1.10 & 2.66
\end{tabular}

$\begin{array}{ccrrr}\text { Pleśna } 2 & & & & \\ \mathrm{E} & \mathrm{Fe}^{3+} & 91.9 & 0.36 & 0.71 \\ & \mathrm{Fe}^{2+} & 8.1 & 1.12 & 2.48\end{array}$

$\begin{array}{ccrcc}\text { Pleśna } 3 & & & & \\ \text { Eg } & \mathrm{Fe}^{3+} & 94.0 & 0.38 & 0.66 \\ & \mathrm{Fe}^{2+} & 6.0 & 1.11 & 2.63\end{array}$

Pleśna 4

$\begin{array}{clrrr}\mathrm{E} & \mathrm{Fe}^{3+} & 91.7 & 0.37 & 0.7 \\ & \mathrm{Fe}^{2+} & 8.3 & 1.12 & 2.5 \\ \mathrm{Eg} & \mathrm{Fe}^{3+} & 93.0 & 0.36 & 0.7 \\ & \mathrm{Fe}^{2+} & 7.0 & 1.12 & 2.49 \\ \text { Łazy } 1 & & & & \\ \mathrm{Eg} & \mathrm{Fe}^{3+} & 90.6 & 0.37 & 0.7 \\ & \mathrm{Fe}^{2+} & 9.4 & 1.13 & 2.5\end{array}$

$\begin{array}{ccrcc}\text { Lazy } 2 & & & & \\ \mathrm{E} & \mathrm{Fe}^{3+} & 91.6 & 0.37 & 0.73 \\ & \mathrm{Fe}^{2+} & 8.4 & 1.15 & 2.49 \\ \text { Lazy 3 } & & & & \\ \mathrm{Eg} & \mathrm{Fe}^{3+} & 92.2 & 0.36 & 0.73 \\ & \mathrm{Fe}^{2+} & 7.8 & 1.12 & 2.44\end{array}$

$\begin{array}{clrrr}\text { Lazy } 4 & & & & \\ \mathrm{E} & \mathrm{Fe}^{3+} & 91.1 & 0.36 & 0.74 \\ & \mathrm{Fe}^{2+} & 8.9 & 1.12 & 2.47 \\ \mathrm{Eg} & \mathrm{Fe}^{3+} & 90.0 & 0.37 & 0.74 \\ & \mathrm{Fe}^{2+} & 10.0 & 1.13 & 2.47\end{array}$

$\begin{array}{ccrrr}\text { Grabie } & & & & \\ \mathrm{Eg} & \mathrm{Fe}^{3+} & 93.1 & 0.36 & 0.72 \\ & \mathrm{Fe}^{2+} & 6.9 & 1.14 & 2.49\end{array}$

\begin{tabular}{|c|c|c|c|c|}
\hline \multicolumn{5}{|c|}{ Radziszów } \\
\hline \multirow[t]{2}{*}{$\mathrm{E}$} & $\mathrm{Fe}^{3+}$ & 92.9 & 0.35 & 0.71 \\
\hline & $\mathrm{Fe}^{2+}$ & 7.1 & 1.10 & 2.47 \\
\hline \multirow[t]{2}{*}{$\mathrm{Eg}$} & $\mathrm{Fe}^{3+}$ & 91.6 & 0.35 & 0.70 \\
\hline & $\mathrm{Fe}^{2+}$ & 8.4 & 1.10 & 2.51 \\
\hline \multicolumn{5}{|c|}{ Polanka Hallera } \\
\hline \multirow[t]{2}{*}{$\mathrm{Eg}$} & $\mathrm{Fe}^{3+}$ & 94.9 & 0.35 & 0.70 \\
\hline & $\mathrm{Fe}^{2+}$ & 5.1 & 1.11 & 2.43 \\
\hline
\end{tabular}

$\begin{array}{ccrrr}\text { Brzezie } & & & & \\ \text { Eg } & \mathrm{Fe}^{3+} & 91.1 & 0.36 & 0.72 \\ & \mathrm{Fe}^{2+} & 8.9 & 1.12 & 2.58\end{array}$

${ }^{\text {a }}$ Contribution of the respective sub-profile to the total absorption profile equivalent to content of $\mathrm{Fe}^{3+}$ and $\mathrm{Fe}^{2+}$

${ }^{\mathrm{b}}$ Isomer shift.

${ }^{\mathrm{c}}$ Quadrupole splitting periodic stagnation of water above the slowly permeable fragipan) on clay mineral transformation in the Retisols. In addition, a prior study (Szymański et al. 2014) indicates that clay minerals in the $\mathrm{E}$ and $\mathrm{Eg}$ horizons of the soils contain mainly organic fillings in their interlayers and not metalhydroxy polymers, as indicated by Brinkman (1979). The neoformation of fine quartz in bleached horizons, proposed by Brinkman $(1977,1979)$ and Brinkman et al. (1973) as a secondary product of weathering due to ferrolysis was not observed in the Retisols, because the presence of quartz in the clay fraction was observed in both $\mathrm{E}$ and $\mathrm{Eg}$ horizons (Fig. 2), and also in other horizons of the soils (Szymański et al. 2011, 2014). In addition, if the ferrolysis leads to the neoformation of fine quartz, and if the process does occur in the Eg horizons, then, the Eg horizons should contain a clearly higher amount of quartz than the E horizons. However, precisely the opposite outcome (i.e., slightly higher mean content of quartz in E versus Eg horizons but not statistically significant at $p<0.05$ ) was identified in the Retisols (Table 5). In addition, the mean chlorite content is the same (Table 5) in the

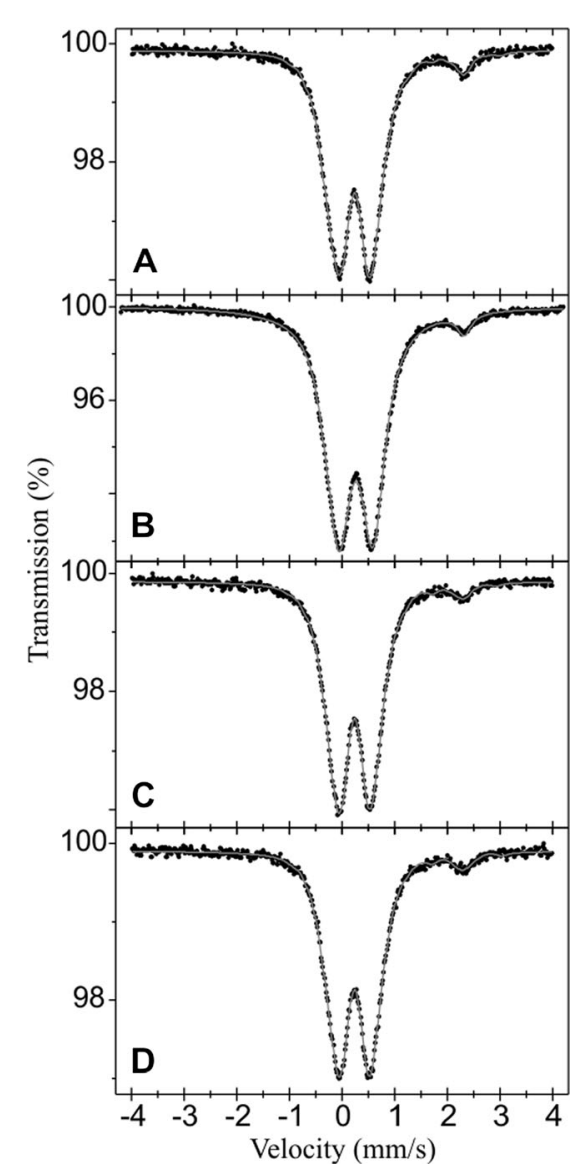

Fig. 6 Mössbauer spectra of the clay fraction $(<2 \mu \mathrm{m})$ from selected eluvial horizons: Eg horizon of the Brzezie profile (a); E horizon of the Pleśna 1 profile (b); E horizon of the Radziszów profile (c); Eg horizon of the Radziszów profile (d) 
E and Eg horizons, but according to Brinkman (1979), the chlorite content should be higher in ferrolyzed horizons in comparison with other horizons. The results of our study are in agreement with Van Ranst and De Coninck (2002) and Van Ranst et al. (2011) who assert that ferrolysis is not the main soil-forming process in bleached soils as in Retisols (Albeluvisols) and Planosols, and it is not responsible for texture contrasts in these soils.

\section{Conclusions}

The obtained results indicate that the qualitative and quantitative mineral compositions of the clay fraction in the $\mathrm{E}$ and $\mathrm{Eg}$ horizons obtained from Retisols in the Carpathian Foothills in Poland exhibiting marked differences in bleaching (strong, moderate, weak, and lack of bleaching) caused by periodic stagnation of water above a slowly permeable fragipan and cyclic redox processes are the same. The E and Eg horizons are characterized by the presence of 2:1 clay minerals with likely organic interlayer fillings, dioctahedral mica, kaolinite, and chlorite. These findings indicate that (1) redox processes occurring in the soils do not affect clay mineral transformation and (2) ferrolysis is not the main soil-forming process operating in these soils. This is most likely because iron-bearing minerals are not abundant in Retisols of the Carpathian Foothills and/or undergo eluviation to the lower part of the soil profiles. The lower content of the clay fraction in the $\mathrm{E}$ and Eg horizons versus that in the lower soil horizons of the Retisols is related to clay illuviation (lessivage), and not to clay decomposition due to ferrolysis.

Acknowledgments This study was financed by Poland's National Science Centre based on Decision Number DEC-2012/05/D/ST10/ 00527. The authors would like to thank Adam Michalski from the Department of Pedology at the Nicolaus Copernicus University in Torun for providing iron and aluminum oxides laboratory data. The authors would like to thank the Editor and anonymous reviewers for helpful suggestions. Language editing was done by Grzegorz Zębik.

Open Access This article is distributed under the terms of the Creative Commons Attribution 4.0 International License (http:// creativecommons.org/licenses/by/4.0/), which permits unrestricted use, distribution, and reproduction in any medium, provided you give appropriate credit to the original author(s) and the source, provide a link to the Creative Commons license, and indicate if changes were made.

\section{References}

Bain DC, Fraser AR (1994) An unusually interlayered clay mineral from the eluvial horizon of a humus-iron podzol. Clay Miner 29:69-76

Barbiero L, Mohan Kumar MS, Violette A, Oliva P, Braun JJ, Kumar C, Furian S, Babic M, Riotte J, Valles V (2010) Ferrolysis induced soil transformation by natural drainage in Vertisols of sub-humid South India. Geoderma 156:173-188
Besson G, Drits VA (1997) Refined relationships between chemical composition of dioctahedral fine-grained mica minerals and their infrared spectra within the $\mathrm{OH}$ stretching region. Part I: identification of the OH stretching bands. Clay Clay Miner 45:158-169

Boivin P, Saejiew A, Grunberger O, Arunin S (2004) Formation of soils with contrasting textures by translocation of clays rather than ferrolysis in flooded rice fields in Northeast Thailand. Eur J Soil Sci 55:713-724

Brinkman R (1970) Ferrolysis, a hydromorphic soil forming process. Geoderma 3(3):199-206

Brinkman R (1977) Surface-water gley soils in Bangladesh: genesis. Geoderma 17(2):111-144

Brinkman R (1979) Ferrolysis, a soil-forming process in hydromorphic conditions. Doctoral thesis. Agricultural Research Reports 887, Centre for Agricultural Publishing and Documentation, Wageningen

Brinkman R, Jongmans AG, Miedema R, Maaskant P (1973) Clay decomposition in seasonally wet, acid soils: micromorphological, chemical and mineralogical evidence from individual argillans. Geoderma 10(4):259-270

Celi L, Schnitzer M, Negre M (1997) Analysis of carboxyl groups in soil humic acids by a wet chemical method, Fourier-transform infrared spectrophotometry, and solution-state carbon-13 nuclear magnetic resonance. A comparative study. Soil Sci 162(3):189-197

Cescas MP, Tyner EH, Harmer IIIRS (1970) Ferromanganiferous soil concretions: a scanning electron microscope study of their micropore structures. Soil Sci Soc Am J 34:641-644

Ciolkosz EJ, Waltman WJ, Thurman NC (1995) Fragipans in Pennsylvania soils. Soil survey. Horizons 36:5-20

Cocozza C, D'Orazio V, Miano TM, Shotyk W (2003) Characterization of solid and aqueous phases of a peat bog profile using molecular fluorescence spectroscopy, ESR and FT-IR, and comparison with physical properties. Organic Geochem 34(1):49-60

Dixon JB, White GN (2002) Manganese oxides. In: Dixon JB, Schulze DG (eds) Soil mineralogy with environmental applications. SSSA Book Ser. 7. Soil Science Society of America Inc., Madison, WI, USA. pp 367-388

Drewnik M, Skiba M, Szymański W, Żyła M (2014) Mineral composition vs. soil forming processes in loess soils - a case study from Kraków (southern Poland. Catena 119:166-173

Ellerbrock RH, Gerke HH (2004) Characterizing organic matter of soil aggregate coatings and biopores by Fourier transform infrared spectroscopy. Eur J Soil Sci 55(2):219-228

Gee GW, Bauder JW (1986) Particle-size analysis. In: Klute A (ed), Methods of soil analysis. Part 1. Physical and mineralogical methods, 2nd edition. Agronomy Monograph. vol. 9. ASA-SSSA, Madison, Wisconsin, pp 427-445

Gerstl Z, Banin A (1980) $\mathrm{Fe}^{2+}-\mathrm{Fe}^{3+}$ transformations in clay and resin ionexchange systems. Clay Clay Miner 28(5):335-345

Hardy M, Jamagne M, Elsass F, Robert M, Chesneau D (1999) Mineralogical development of the silt fractions of a Podzoluvisol on loess in the Paris Basin (France. Eur J Soil Sci 50:443-456

Hess M (1965) Altitudinal climatic zones in the Polish Western Carpathians. Zeszyty. Naukowe UJ Prace Geograficzne 11 (in Polish)

Hobson, WA, Dahlgren, RA (1998) Soil forming processes in vernal pools of Northern California, Chico Area. In: Witham CW, Bauder ET, Belk D, Ferren Jr. WR, Ornduff R (eds) Ecology, conservation, and management of vernal pool ecosystems - proceedings from a 1996 conference. California Native Plant Society, Sacramento, CA, pp 24-37

IUSS Working Group WRB (2014) World reference base for soil resources 2014. World Soil Resources Reports No. 106. FAO, Rome

Jackson ML (1969) Soil chemical analysis - advance course, 2nd edn. University of Wisconsin, Madison, WI

Kalinina O, Chertov O, Dolgikh AV, Goryachkin SV, Lyuri DI, Vormstein S, Giani L (2013) Self-restoration of post-agrogenic Albeluvisols: 
soil development, carbon stocks and dynamics of carbon pools. Geoderma 207-208:221-233

Kemp RA, McIntosh PD (1989) Genesis of a texturally-banded soil in Southland, New Zealand. Geoderma 85:65-81

Klimaszewski M (1967) The polish western Carpathians in quaternary period. In: Galon R, Dylik J (eds) . Quaternary of Poland, PWN, Warszawa, pp. 431-497 in Polish

Kohut C, Warren CJ (2002) Chlorite. In: Dixon JB, Schulze DG (eds) Soil mineralogy with environmental applications. Soil Sci. Soc. Am, Madison, WI, pp. 531-556

Leigh DS (1998) Evaluating artifact burial by eolian versus bioturbation processes, South Carolina Sandhills, USA. Geoarchaeology 13: 309-330

Lindbo DL, Veneman PLM (1993) Micromorphology of selected Massachusetts fragipan soils. Soil Sci Soc Am J 57:437-442

Lindbo DL, Rhoton FE, Bigham JM, Hudnall WH, Jones FS, Smeck NE, Tyler DD (1994) Bulk density and fragipan identification in loess soils of the lower Mississippi River valley. Soil Sci Soc Am J 58: 884-891

Lindbo DL, Rhoton FE, Bigham JM, Hudnall WH, Jones FS, Smeck NE, Tyler DD (1995) Loess toposequences in the lower Mississippi River valley: I. Fragipan morphology and identification. Soil Sci Soc Am J 59:487-500

Lindbo DL, Rhoton FE, Hudnall WH, Smeck NE, Bigham JM, Tyler DD (2000) Fragipan degradation and nodule formation in Glossic Fragiudalfs of the lower Mississippi River valley. Soil Sci Soc Am J 64:1713-1722

Madejová J (2003) FTIR techniques in clay mineral studies. Vib Spectrosc 31:1-10

Montagne D, Cornu S, Le Forestier L, Hardy M, Josière O, Caner L, Cousin I (2008) Impact of drainage on soil-forming mechanisms in a French Albeluvisol: input of mineralogical data in massbalance modelling. Geoderma 145:426-438

Moore D, Reynolds RC (1997) X-ray diffraction and the identification and analysis of clay minerals. Oxford University Press, Oxford

Mystkowski K (1999) ClayLab, a computer program for processing and interpretation of x-ray diffractograms of clays. Conference of European Clay Groups Association, EUROCLAY 1999, Book of Abstracts, Kraków, Poland, pp 114-115

Nakao A, Funakawa S, Kosaki T (2009) Hydroxy-Al polymers block the frayed edge sites of illitic minerals in acid soils: studies in southwestern Japan at various weathering stages. Eur J Soil Sci 60:127-138

Nelson DW, Sommers LE (1996) Total carbon, organic carbon, and organic matter. In: Sparks DL, Page AL, Helmke PA et al. (eds) Methods of soil analysis. Part 3. Chemical methods SSSA Book Series. vol. 5. SSSA and ASA, Madison, Wisconsin, pp 961-1010

Nikorych VA, Szymański W, Polchyna SM, Skiba M (2014) Genesis and evolution of the fragipan in Albeluvisols in the Precarpathians in Ukraine. Catena 119:154-165

Peacock E, Fant DW (2002) Biomantle formation and artifact translocation in upland sandy soils, an example from the Holly Springs National Forest, north-central Mississippi, U.S.A. Geoarchaeology 17:91-114

Phillips JD (2001) Contingency and generalization in pedology, as exemplified by texture-contrast soils. Geoderma 102:347-370

Phillips JD (2004) Geogenesis, pedogenesis, and multiple causality in the formation of texture-contrast soils. Catena 58:275-295

Phillips JD (2007) Development of texture contrast soils by a combination of bioturbation and translocation. Catena 70:92-104

Rhoton FE, Bigham JM, Schulze DG (1993) Properties of ironmanganese nodules from a sequence of eroded fragipan soils. Soil Sci Soc Am J 57:1386-1392

Rich CI (1968) Hydroxy interlayers in expansible layer silicates. Clay Clay Miner 16:15-30
Rozenson I, Heller-Kallai L (1978) Reduction and oxidation of $\mathrm{Fe}^{3+}$ in dioctahedral smectites - III. Oxidation of octahedral iron in montmorillonite. Clay Clay Miner 26(2):88-92

Sauer D, Schülli-Maurer I, Sperstad R, Sørensen R (2013) Micromorphological characteristics reflecting soil-forming processes during Albeluvisol development in S Norway. Spanish. J Soil Sci 3(2):38-58

Schlichting E, Blume HP (1966) Bodenkundliches Praktikum. Verlag Paul Parey, Hamburg-Berlin

Schwertmann U, Fanning DS (1976) Iron-manganese concretions in hydrosequences of soils in loess in Bavaria. Soil Sci Soc Am J 40: 731-738

Skiba S, Drewnik M (2003) Soil map of the polish Carpathians. Roczniki Bieszczadzkie 11:15-20 in Polish

Środoń J (2006) ) Identification and quantitative analysis of clay minerals. In: Bergaya $\mathrm{F}$ et al. (eds) Handbook of clay science. Elsevier, Amsterdam, pp. 765-787

Starkel L (1972) The Outer Carpathians. In: Klimaszewski M (ed) Geomorphology of Poland, Southern Poland, mountains and uplands, PWN, Warszawa, pp 55-115 (in Polish)

Świtoniak M (2008) Classification of young glacial soils with vertical texture-contrast using WRB system. Agrochimija i Gruntoznawstwo 69:96-101

Świtoniak M (2014) Use of soil profile truncation to estimate influence of accelerated erosion on soil cover transformation in young morainic landscapes, north-eastern Poland. Catena 116:173-184

Świtoniak M, Mroczek P, Bednarek R (2016) Luvisols or Cambisols? Micromorphological study of soil truncation in young morainic landscapes - case study: Brodnica and Chełmno Lake districts (North Poland. Catena 137:583-595

Szymański W, Skiba M (2013) Distribution, morphology, and chemical composition of Fe-Mn nodules in Albeluvisols of the Carpathian foothills, Poland. Pedosphere 23(4):445-454

Szymański W, Skiba M, Skiba S (2011) Fragipan horizon degradation and bleached tongues formation in Albeluvisols of the Carpathian Foothills, Poland. Geoderma 167-168:340-350

Szymański W, Skiba M, Skiba S (2012) Origin of reversible cementation and brittleness of the fragipan horizon in Albeluvisols of the Carpathian foothills, Poland. Catena 99:66-74

Szymański W, Skiba M, Nikorych VA, Kuligiewicz A (2014) Nature and formation of interlayer fillings in clay minerals in Albeluvisols from the Carpathian foothills, Poland. Geoderma:235-236 396-409

Taut T, Kleeberg R, Bergmann J (1998) The new Seifert Rietveld program BGMN and its application to quantitative phase analysis. Materials Structure 5(1):57-64

Thomas GW (1996) Soil pH and soil acidity. In: Sparks DL, Page AL, Helmke PA, Loeppert RH (eds) Methods of soil analysis. Part 3. Chemical methods SSSA book series, 5 edn. SSSA and ASA, Madison, Wisconsin, pp. 475-490

Tivet F, Sá JCM, Lal R, Milori DMBP, Briedis C, Letourmy P, Pinheiro LA, Borszowskei PR, Hartman DC (2013) Assessing humification and organic $\mathrm{C}$ compounds by laser-induced fluorescence and FTIR spectroscopies under conventional and no-till management in Brazilian Oxisols. Geoderma 207-208:71-81

Towpasz K, Zemanek B (1995) Vegetation. In: Warszyńska J (ed), Polish Carpathian Mts.-Environment, Man, and His Activity. Wyd. UJ, Kraków, pp 77-94 (in Polish)

Van Ranst E, De Coninck F (2002) Evaluation of ferrolysis in soil formation. Eur J Soil Sci 53:513-519

Van Ranst E, Dumon M, Tolossa AR, Cornelis JT, Stoops G, Vandenberghe RE, Deckers J (2011) Revisiting ferrolysis processes in the formation of Planosols for rationalizing the soils with stagnic properties in WRB. Geoderma 163:265-274

Van Reeuwijk LP (2002) Procedures for soil analysis, 6th edn. ISRIC, Wageningen 
Weisenborn BN, Schaetzl RJ (2005) Range of fragipan expression in some Michigan soils: I. Morphological, micromorphological, and pedogenic characterization. Soil Sci Soc Am J 69:168-177

Witty JE, Knox EG (1989) Identification, role in soil taxonomy and worldwide distribution of fragipans. In: Smeck NE, Ciolkosz EJ (eds) Fragipans: their occurrence, classification and genesis. SSSA Spec. Publ., 24. SSSA, Madison, Wisconsin, pp 1-9

Zasoński S (1983) Main soil-forming processes on loess-like deposits of the Wieliczka foothills. Part II. Micromorphological properties. Roczniki Gleboznawcze 34(4):123-161 in Polish
Zasoński S (1992) Micromorphological properties of silty soils of the Krosno Valley. Zeszyty Naukowe AR 265:19-33 in Polish

Zhang M, Karathanasis AD (1997) Characterization of iron-manganese concretions in Kentucky Alfisols with perched water tables. Clay Clay Miner 45:428-439

Zviagina BB, McCarty DK, Środoń J, Drits VA (2004) Interpretation of infrared spectra of dioctahedral smectites in the region of $\mathrm{OH}-$ stretching vibrations. Clay Clay Miner 52: $399-410$ 